\title{
Nano materyal içerikli katkıların yakıt özelliklerine ve motor performansina etkileri
}

\author{
İsmet SEZER ${ }^{*}$ \\ Gümüşhane Üniversitesi, Makine Mühendisliği Bölümü, 29100 Gümüşhane, Turkey \\ Geliş Tarihi (Recived Date): 02.03.2018 \\ Kabul Tarihi (Accepted Date): 14.06.2018
}

\section{Özet}

Bu çalışma; dizel, biyodizel, su içeren emülsiyon yakıtları ve çeşitli yakıt karışımları gibi dizel motor yakıtlarında nano materyal içerikli katkıların kullanılmasının motor performansı ve emisyonlara etkileri üzerine yapılmış çeşitli çalışmaların sonuçlarından derlenmiştir. Dizel motorlarda zararlı egzoz emisyonlarını azaltması için üç farklı teknik uygulanmaktadır. Zararlı emisyonları azaltmak için uygulanan tekniklerden ilki motor tasarımda ve yakıt püskürtme sisteminde değişiklikler yapılarak yanmanın iyileştirilmesidir. Ancak bu pahal ve zaman alıc bir süreçtir. İkinci teknik katalitik konvertör ve partikül filtresi gibi çeşitli egzoz gazı azaltıcı sistemler kullanmaktır. Ancak, bu sistemler dizel motorun performansinı olumsuz yönde etkiler. Dizel motorlarda, emisyonları azaltmak ve aynı zamanda dizel motorun performansinı artırmak için kullanılan üçüncü teknik ise çeşitli yakıt katkılarının kullanılmasıdır. Dizel motorlardan yayllan en önemli kirleticiler azot oksit (NOx) ve partikül madde (PM-is) emisyonlarıdır. Uygulamada, NOx ve PM emisyonlarını birlikte azaltmak oldukça zordur. Emisyonlarl azaltmanın ve motor performansinı artırmanin en iyi yolunun nano materyal içerikli katkıların ve suyla emülsiyonlaştırılmış yakıtların kullanımı olduğu birçok araştırmacı tarafindan bildirilmektedir. Bu derlemede çeşitli dizel motor yakıtlarında farklı nano materyal içerikli katkıların kullanımının yakıt özellikleri ve motor performansina etkileri incelenmiştir.

Anahtar kelimeler: Dizel motor performansı, biyodizel, emülsiyon yakıt, nano materyal katkılar.

\footnotetext{
* İsmet SEZER, isezer@gumushane.edu.tr, https://orcid.org/0000-0001-7342-9172
} 


\title{
Effect of additives including nano materials on fuel properties and engine performance
}

\begin{abstract}
This study compiled the results of various researches performed on the effects of performance and emissions of diesel engine using nanomaterials additives in diesel engine fuels such as diesel, biodiesel, water emulsified fuels and various fuel blends. Three different techniques are used in reduction of the harmful exhaust emissions of the diesel engine. The first technique for the reduction of harmful emissions is improving the combustion by modification of engine design and fuel injection system, but this process is expensive and time consuming. The second technique is the using various exhaust gas devices like catalytic converter and diesel particulate filter. However, the use of these devices affects negatively diesel engine performance. The other technique to reduce emissions and also improve diesel engine performance is the use of various fuel additives. The major pollutants of diesel engine are oxide of nitrogen (NOx) and particulate matter (PM). It is very difficult to reduce NOx and PM simultaneously in practice. The most researches declare that the best way to reduce the emissions and increase the engine performance is the use of nano material additives and water emulsified fuels. The effects of using different nano material additives in various diesel engine fuels on the fuel properties and engine performance were investigated in this review.
\end{abstract}

Keywords: Diesel engine performance, biodiesel, emulsified fuel, nano material additives.

\section{Giriş}

Dizel motorların; karayolu taşıtlarında, tarım sektöründe, elektrik jeneratörlerinde güç kaynağı olarak kullanımının sürekli artması yakıt tüketiminin ve fiyatının sürekli artışına neden olmaktadır. Diğger taraftan, emisyonlarla ilgili sıkı düzenlemeler emisyon azaltma teknolojilerinin ve alternatif yakıtların kullanılmasını zorunlu k1lmaktadır. Katalitik konvertör ve partikül filtresi kullanımı dizel motorlarda azot oksit $\left(\mathrm{NO}_{\mathrm{x}}\right)$ ve partikül madde (PM-is) emisyonlarını azaltması yanında motor performansını ve yakıt ekonomisini olumsuz yönde etkilemektedir. Dizel motorların performansını artırıp emisyonlarını azaltmanın diğer bir yolu ise özellikle gıda olarak tüketilmeyen yağlardan üretilen biyodizel yakıtların kullanılmasıdır [1]. Ayrıca, biyodizel yakıtların dizel yakıtına belirli oranlarda katılarak kullanılmasının karbondioksit $\left(\mathrm{CO}_{2}\right)$ salımını azaltarak küresel 1sınmayı azaltabileceği düşünülmektedir [2]. Yapılan çeşitli deneysel çalışmalar biyodizel yakıt ve karışımlarının motor gücünde bir miktar düşüşe ve yakıt tüketiminde artışa neden olduğunu göstermiştir. Ayrıca, biyodizel yakıt ve karışımlarının içeriğindeki oksijen nedeniyle $\mathrm{NO}_{\mathrm{x}}$ emisyonunda artış olduğu bildirilmektedir [3-4]. Biyodizel yakıtların bu olumsuz etkilerinin emülsiyon yöntemi ve çeşitli yakıt katkıları kullanılarak azaltılması mümkündür. Dizel ve biyodizel yakıtların su ile emülsiyonlaştırılmasının $\mathrm{NO}_{\mathrm{x}}$ emisyonunun azaltılmasında oldukça etkili bir yöntem olduğu belirtilmektedir [5-6]. Ancak, emülsiyon yakıtların içinde bulunan su yanma sıcaklığını düşürerek tutuşma gecikmesi süresinin uzamasına neden olabilmektedir [7]. Bu olumsuz etkiyi azaltmak için suyla emülsiyonlaştırılmış 
yakıtlarda nano materyal içerikli katkılarının kullanılması önerilmektedir [8-9]. Bu nedenle, dizel, biyodizel ve suyla emülsiyonlaştırılmış yakıtlarda nano materyal katkıların kullanımı üzerine yapılan çalışmaların sonuçlarının bir arada değerlendirilmesi motor performansının iyileştirilmesi ve emisyonlarının azaltılması açısından oldukça önemlidir. Sunulan çalışmada dizel yakıtı, çeşitli biyodizel yakıt ve karışımları ile suyla emülsiyonlaştırılmış yakıtlarda nano materyal katkıların kullanılmasının yakıt özellikleri ve motor performansına etkileri litertüre dayalı olarak incelenmiştir.

\section{Nano materyal içerikli yakıt katkıları}

Nano materyallerin boyutları 1-100 nm arasında değişmekte olup yakıt katkısı olarak kullanılan nano materyaller arasında $\mathrm{Al}, \mathrm{Ag}, \mathrm{Cu}, \mathrm{Mg}, \mathrm{Ni}, \mathrm{Ti}, \mathrm{Zn}$ gibi metaller, bor gibi yarı metaller ve $\mathrm{Al}_{2} \mathrm{O}_{3}, \mathrm{CeO}_{2}, \mathrm{TiO}_{2}, \mathrm{Fe}_{2} \mathrm{O}_{3} \mathrm{Fe}_{3} \mathrm{O}_{4} \mathrm{CuO}, \mathrm{MnO}$, MgO gibi metal oksit nano akışkanlar sıralanabilir [10-11]. Son zamanlarda, nano boyutlu silikon tozları ve nano gözenekli silikonlu levhalar ile tek veya çok tabakalı nano tüpler gibi materyaller de enerji uygulamalarında kullanılmaktadır [12]. Literatürde nano materyallerin yakıt katkısı olarak kullanıldığı birçok çalışma bulunmaktadır. Sonawane $v d$ [13] havacılık türbin yakıtına (Kerosin-K) alüminyum oksit $\left(\mathrm{Al}_{2} \mathrm{O}_{3}\right)$ katılmasının etkilerini incelemişlerdir. Dizel (D) yakıtına, nano alüminyum ( $\mathrm{n}-\mathrm{Al}$ ), nano gümüş ( $\mathrm{n}-\mathrm{Ag}$ ), nano seryum ( $\mathrm{n}-\mathrm{Ce}$ ), nano platin ( $\mathrm{n}-\mathrm{Pt}$ ), nano demir ( $\mathrm{n}-\mathrm{Fe}$ ), nano bor (n-B) gibi nano parçacıkların katılmasının etkileri çeşitli araştırmacılarca [14-20] incelenmiştir. Dizel yakıtına, $\mathrm{Al}_{2} \mathrm{O}_{3}$, çinko oksit $(\mathrm{ZnO})$, demir-II oksit $\left(\mathrm{Fe}_{2} \mathrm{O}_{3}\right)$ ve demir-III oksit $\left(\mathrm{Fe}_{3} \mathrm{O}_{4}\right)$, bakır oksit $(\mathrm{CuO})$, kobalt oksit $\left(\mathrm{Co}_{3} \mathrm{O}_{4}\right)$, titanyum oksit $\left(\mathrm{TiO}_{2}\right)$, grafit oksit $(\mathrm{GO})$, seryum oksit $\left(\mathrm{CeO}_{2}\right)$, mangan oksit $(\mathrm{MnO})$, mangan dioksit $\left(\mathrm{MnO}_{2}\right)$, magnezyum oksit (MgO), kalsiyum oksit (CaO) gibi nano akışkanlar katılmasının etkileri çeşitli araştırmacılarca [21-46] incelenmiştir. Dizel yakıtına karbon nano tüpler (KNT) ve çok tabakalı karbon nano tüpler (ÇTKNT) katılmasının etkileri Aalam vd [47] tarafından incelenmiştir. Farklı oranlarda su içeren dizel emülsiyon yakıtlarına farklı nano materyaller katılmasının etkileri çeşitli araştırmacılarca [48-52] incelenmiştir. Farklı oranlarda etanol (E) içeren dizel yakıtlarına farklı türde nano materyaller katılmasının etkileri çeşitli araştırmacılarca [53-55] incelenmiştir. Farklı türden biyodizel yakıtlara farklı türde nano materyal içerikli katkıların katılmasının etkileri çeşitli araştırmacılar tarafından [41, 56-73] incelenmiștir. Farklı türden ve farklı oranlarda su veya etanol içeren biyodizel emülsiyon yakıtlarda farklı türde nanomateryal içerikli katkılar kullanılmasının etkiler çeşitli araştırmacılarca [74-78] incelenmiştir. Farklı türden biyodizel-dizel yakıt karışımlarına farklı türden nano materyal içerikli katkılar katılmasının etkileri birçok araştırmacı tarafından [12, 38, 45, 79-114] incelenmiştir.

\section{Nano materyal içerikli katkıların yakıt özelliklerine etkileri}

Nano materyal katkı maddeleri, arttırılmış yüzey alanı/hacim oranı, hızlı buharlaşma ve daha kısa tutuşma gecikmesi süresi gibi özellikleri nedeniyle yakıt özelliklerini iyileştirmek için uygun bir katalizör olarak düşünülmektedir [115]. Nano materyal içerikli katkıların yoğunluk, viskozite, alevlenme ve tutuşma noktası sıcaklıkları, akma ve bulutlanma noktası sıcaklıkları, setan sayısı ve 1sıl değer gibi yakıt özelliklerine etkisi birçok araştırmacı tarafından incelenmiştir. Tablo 1'de çeşitli nano materyal katkıların yakıt özelliklerine etkilerine yönelik sayısal değerler verilmiştir. 
Tablo 1. Nanomateryal içerikli katkıların yakıt özelliklerine etkileri.

\begin{tabular}{|c|c|c|c|c|c|c|c|}
\hline Ana yakıt+katk1 & $\begin{array}{c}\text { Yoğunluk } \\
\text { (\%değişim) }\end{array}$ & $\begin{array}{l}\text { Viskozite } \\
\text { (\%değişim) }\end{array}$ & $\begin{array}{l}\text { Alevlenme } \\
\text { Sicaklığı } \\
\text { (\%değişim) }\end{array}$ & $\begin{array}{c}\text { Tutuşma } \\
\text { Sicaklığı } \\
\text { (\%değişim) }\end{array}$ & $\begin{array}{l}\text { Setan Sayısı } \\
\text { (\%değişim) }\end{array}$ & $\begin{array}{l}\text { Isıl değer } \\
\text { (\%değişim) }\end{array}$ & Kaynak \\
\hline $\mathrm{K}+\% 0,1-1 \mathrm{Al}_{2} \mathrm{O}_{3}$ & - & $\uparrow 2-37$ & - & - & - & - & [13] \\
\hline $\mathrm{D}+25-75 \mathrm{ppm}$ n-Al & $\downarrow 4,7-12,5$ & $\rightarrow$ & $\uparrow 3,6-12,7$ & - & - & - & [15] \\
\hline $\mathrm{D}+25-50 \mathrm{ppm} \mathrm{Al}_{2} \mathrm{O}_{3}$ & $\uparrow 0,2-0,3$ & $\uparrow 1,5-2,7$ & $\uparrow 4,1-8,3$ & $\uparrow 3,8-7,7$ & - & $\uparrow 0,3-0,6$ & {$[22]$} \\
\hline $\mathrm{D}+250-1000 \mathrm{ppm} \mathrm{Al} \mathrm{O}_{3}$ & $\uparrow 0,2-1,1$ & - & $\uparrow 1,9-15,4$ & $\uparrow 4,8-22,6$ & - & $\uparrow 0,09-0,6$ & [23] \\
\hline $\mathrm{D}+150-300 \mathrm{mg} / \mathrm{L} \mathrm{Fe}_{3} \mathrm{O}_{4}$ & $\uparrow 0,3-0,6$ & $\uparrow 3,7-11,1$ & $\downarrow 10,9-14,5$ & $\downarrow 5-17$ & $\uparrow 4,2-10,6$ & - & [27] \\
\hline $\mathrm{D}+25-50 \mathrm{ppm} \mathrm{Fe} \mathrm{O}_{3}$ & $\uparrow 0,5-1$ & - & $\uparrow 8,6-15,5$ & - & $\uparrow 3-5,5$ & $\uparrow 0,8-1,5$ & [29] \\
\hline $\mathrm{D}+25-100 \mathrm{ppm} \mathrm{Al}_{2} \mathrm{O}_{3}$ & $\uparrow 0,08-0,12$ & $\downarrow 0-2,8$ & $\uparrow 10-15$ & - & $\uparrow 0,9-1,5$ & - & [30] \\
\hline $\mathrm{D}+50 \mathrm{ppm} \mathrm{CuO}$ & $\uparrow 0,07$ & $\downarrow 2,8$ & $\uparrow 10$ & - & $\uparrow 1,3$ & - & [30] \\
\hline $\mathrm{D}+300 \mathrm{ppm} \mathrm{Al}_{2} \mathrm{O}_{3}$ & $\uparrow 0,1$ & $\downarrow 2,8$ & $\downarrow 5,7$ & - & - & $\uparrow 0,04$ & [32] \\
\hline $\mathrm{D}+300 \mathrm{ppm} \mathrm{TiO}_{2}$ & $\uparrow 0,09$ & $\downarrow 2,8$ & $\downarrow 1,9$ & - & - & $\uparrow 0,005$ & [32] \\
\hline $\mathrm{D}+\% 0,01-0,1 \mathrm{GO}$ & $\downarrow 3,1 \uparrow 0,03$ & $\downarrow 31-8,6$ & $\downarrow 14,4-4,8$ & - & $\downarrow 2,2-13$ & - & [32] \\
\hline $\mathrm{D}+\% 0,01-0,1 \mathrm{Al}_{2} \mathrm{O}_{3}$ & $\downarrow 3,1 \uparrow 0,09$ & $\downarrow 32,5 \uparrow 6,5$ & $\downarrow 16,9-1,2$ & - & $\downarrow 6,5-13$ & - & [33] \\
\hline $\mathrm{D}+\% 0,01-0,1 \mathrm{CeO}_{2}$ & $\downarrow 3,1 \uparrow 0,1$ & $\downarrow 33,1-0,6$ & $\downarrow 18-12$ & - & $\downarrow 8,7-10,8$ & - & [33] \\
\hline $\mathrm{D}+200 \mathrm{mg} / \mathrm{L} \mathrm{CuO}$ & - & $\downarrow 17$ & $\downarrow 20$ & $\downarrow 14,8$ & - & - & [34] \\
\hline $\mathrm{D}+200 \mathrm{mg} / \mathrm{L} \mathrm{MnO}$ & - & $\downarrow 6,3$ & $\downarrow 8,3$ & $\downarrow 3,7$ & - & - & [34] \\
\hline $\mathrm{D}+8-16 \mu \mathrm{mol} / \mathrm{L} \mathrm{MnO}$ & - & $\downarrow 3,8-11,5$ & $\downarrow 6,8-12,3$ & - & - & - & [35] \\
\hline $\mathrm{D}+8-16 \mu \mathrm{mol} / \mathrm{L} \mathrm{MgO}$ & - & $\downarrow 7,7-15,4$ & $\downarrow 5,5-10,9$ & - & - & - & [35] \\
\hline $\mathrm{D}+54,2 \mu \mathrm{mol} / \mathrm{L} \mathrm{MnO}$ & - & $\downarrow 5,2$ & $\downarrow 5,3$ & - & $\uparrow 4,4$ & - & {$[36]$} \\
\hline $\mathrm{D}+50 \mathrm{cc} / \mathrm{L} \mathrm{CeO}_{2}$ & $\uparrow 0,2$ & - & $\uparrow 14,8$ & $\uparrow 16,1$ & - & $\uparrow 0,36$ & [39] \\
\hline $\mathrm{SBBD}+25 \mathrm{ppm} / \mathrm{L} \mathrm{CeO}_{2}$ & $\uparrow 0,4$ & $\uparrow 4,4$ & $\uparrow 229,3$ & $\uparrow 172$ & $\uparrow 24,4$ & $\uparrow 21,3$ & [41] \\
\hline $\mathrm{D}+50 \mathrm{cc} / \mathrm{L} \mathrm{CeO}_{2}$ & $\uparrow 0,2$ & - & $\uparrow 14,8$ & $\uparrow 16,1$ & - & $\uparrow 0,36$ & [45] \\
\hline $\mathrm{D}+\% 0,2 \mathrm{TiO}_{2}$ & $\uparrow 0,02$ & $\uparrow 2,2$ & $\uparrow 4,4$ & $\rightarrow$ & - & - & {$[46]$} \\
\hline D+25-50 ppm ÇTKNT & $\uparrow 0,5-0,98$ & - & $\uparrow 5,2-12$ & - & $\uparrow 2,9-5,5$ & $\uparrow 1-1,7$ & [47] \\
\hline DW15S2+25-100 ppm n-Al & $\uparrow 0,07-0,13$ & $\uparrow 0,8-2,2$ & $\uparrow 1,6-4,8$ & - & $\uparrow 11,6-16,3$ & $\uparrow 1,3-2,8$ & [48] \\
\hline DW1+\%0,1 n-Al & $\uparrow 1,7$ & $\uparrow 19,3$ & - & - & $\uparrow 7,8$ & $\downarrow 1,3$ & [49] \\
\hline DW1+\%0,1 n-Si & $\uparrow 1,4$ & $\uparrow 20,2$ & - & - & $\uparrow 11,7$ & $\downarrow 1,3$ & [49] \\
\hline DW15S2+ 50-150 ppm ÇTKNT & $\uparrow 3-3,7$ & $\uparrow 83,5-91,2$ & $\uparrow 17,2-24,1$ & $\uparrow 11,3-19,7$ & $\uparrow 2,1-6$ & $\downarrow 9,3-4,6$ & [50] \\
\hline DW20S2+ 50-150 ppm ÇTKNT & $\uparrow 4-4,5$ & $\begin{array}{c}\uparrow 104,8- \\
109,9\end{array}$ & $\uparrow 18,9-36,2$ & 个16,9-29,6 & $\uparrow 0-4,2$ & $\downarrow 9,3-6,5$ & {$[51]$} \\
\hline $\mathrm{JBD}+30 \mathrm{ppm} / \mathrm{L} \mathrm{CeO}_{2}$ & $\uparrow 0,3$ & $\uparrow 4,8$ & $\downarrow 10,6$ & - & - & $\downarrow 2$ & [52] \\
\hline $\mathrm{JBD}+25-50 \mathrm{ppm} \mathrm{n-Al}$ & $\uparrow 0,1-0,2$ & $\uparrow 1,1-1,9$ & $\downarrow 1,2-3,5$ & - & $\uparrow 1,9-3,8$ & $\uparrow 0,9-1,7$ & {$[56]$} \\
\hline JBD+25-50 ppm KNT & $\uparrow 0,05-0,3$ & $\uparrow 0,7-1,5$ & $\downarrow 2,3-4,7$ & - & $\uparrow 3,8-7,5$ & $\uparrow 1,6-2,3$ & {$[56]$} \\
\hline $\mathrm{HBD}+50 \mathrm{ppm}$ n-Ag & $\uparrow 2,8$ & $\uparrow 5,3$ & $\downarrow 1,2$ & - & - & $\downarrow 2,9$ & [58] \\
\hline $\mathrm{HBD}+50 \mathrm{ppm}$ n-G & $\uparrow 2,3$ & $\uparrow 3,6$ & $\downarrow 7$ & - & - & $\downarrow 1,4$ & [58] \\
\hline HBD+50 ppm ÇTKNT & $\uparrow 2,3$ & $\uparrow 3,6$ & $\downarrow 3,5$ & - & - & $\downarrow 2,5$ & [58] \\
\hline HBD+25-50 ppm n-Ag & $\uparrow 2,3-3,4$ & $\uparrow 3,6$ & $\downarrow 14,4-15,5$ & - & - & $\downarrow 3-1,7$ & [59] \\
\hline $\mathrm{HBD}+25-50 \mathrm{ppm} \mathrm{n-G}$ & $\uparrow 1,7-2,3$ & $\uparrow 3,6$ & $\downarrow 5,9-7$ & - & - & $\downarrow 2,8-1,4$ & {$[60]$} \\
\hline PAMBD+4-16 $\mu \mathrm{mol} / \mathrm{L} \mathrm{n}-\mathrm{Mn}$ & $\downarrow 0,7-2,5$ & $\downarrow 3,2-9,1$ & $\downarrow 2,3-8$ & - & - & $\uparrow 2,2-2,8$ & [61] \\
\hline $\mathrm{JBD}+30 \mathrm{ppm} / \mathrm{L} \mathrm{Al}_{2} \mathrm{O}_{3}$ & $\uparrow 0,2$ & $\uparrow 3,6$ & $\downarrow 8,2$ & - & - & $\downarrow 1,5$ & [62] \\
\hline $\mathrm{NBD}+100-300 \mathrm{ppm} \mathrm{Al}_{2} \mathrm{O}_{3}$ & $\uparrow 0,1-0,2$ & $\uparrow 0,2-0,4$ & $\uparrow 0,5-1,1$ & - & $\uparrow 0-1,9$ & $\uparrow 0,5-0,6$ & [63] \\
\hline $\mathrm{ATBD}+5-50 \mu \mathrm{mol} / \mathrm{L} \mathrm{FeCl}_{3}$ & $\downarrow 0,02-0,1$ & $\downarrow 0,2-1,1$ & $\downarrow 0-2,9$ & $\downarrow 0-3,7$ & $\uparrow 2,1-5,4$ & $\uparrow 0,2-1$ & [64] \\
\hline $\mathrm{MBD}+\% 1 \mathrm{Fe}_{3} \mathrm{O}_{4}$ & $\uparrow 5$ & $\uparrow 3,7$ & $\uparrow 2,1$ & - & $\uparrow 8$ & $\uparrow 2,8$ & [66] \\
\hline $\mathrm{NBD}+30 \mathrm{ppm} / \mathrm{L} \mathrm{CeO}_{2}$ & $\downarrow 2$ & $\downarrow 24,5$ & $\downarrow 3,9$ & - & $\uparrow 0,9$ & $\uparrow 3,9$ & {$[70]$} \\
\hline NBD+100-300 ppm KNT & $\downarrow 0,11-0,22$ & $\uparrow 0,2-0,4$ & $\uparrow 0,5-1,1$ & - & $\uparrow 0-1,9$ & $\uparrow 0,5-0,6$ & {$[71]$} \\
\hline AYBD+100ppm KNT & $\uparrow 9,6$ & $\uparrow 13,4$ & $\uparrow 40$ & $\uparrow 29,3$ & $\uparrow 5,9$ & $\downarrow 0,97$ & {$[72]$} \\
\hline HBD+25-50 ppm ÇTKNT & $\uparrow 2-2,3$ & $\uparrow 1,8-3,6$ & $\downarrow 2,3-3,5$ & - & - & $\downarrow 4-2,5$ & [73] \\
\hline LOBDW5S2+30 ppm $\mathrm{CeO}_{2}$ & $\uparrow 1,1$ & $\uparrow 6,8$ & $\downarrow 9,5$ & - & $\uparrow 5,4$ & $\uparrow 1,1$ & {$[76]$} \\
\hline JBDW5S2+25-100 ppm KNT & $\downarrow 0,3-0,04$ & $\uparrow 0,5-9,4$ & $\downarrow 7,1-12,8$ & - & $\uparrow 5,9-9,8$ & $\uparrow 0,6-2,1$ & [77] \\
\hline DZJBD25+25-50 ppm $\mathrm{Al}_{2} \mathrm{O}_{3}$ & $\uparrow 0,3-0,8$ & $\downarrow 4,8-10,9$ & $\uparrow 1,8-3,6$ & - & $\uparrow 3,6-5,4$ & - & [82] \\
\hline DUCBD20+50-100 $\mathrm{ppm} \mathrm{Al}_{2} \mathrm{O}_{3}$ & $\uparrow 1,3-2,7$ & $\uparrow 1,7$ & $\uparrow 67-77$ & - & $\uparrow 4,3-6,5$ & $\uparrow 4,4-6,9$ & [83] \\
\hline DPAMBDX+50-100 ppm $\mathrm{Al}_{2} \mathrm{O}_{3}$ & $\uparrow 0,8-1,4$ & - & - & - & - & - & {$[84]$} \\
\hline DSBDX+50-100 ppm $\mathrm{Al}_{2} \mathrm{O}_{3}$ & $\uparrow 0,3$ & - & - & - & - & - & [85] \\
\hline DDBDX+100 ppm $\mathrm{Al}_{2} \mathrm{O}_{3}$ & $\uparrow 0,3-0,8$ & $\uparrow 0,6-1,1$ & $\uparrow 4,1-5,1$ & $\uparrow 4-5$ & - & $\downarrow 0,7-2,3$ & {$[86]$} \\
\hline DMBD20+50 ppm CuO & $\downarrow 1$ & $\downarrow 8,5$ & $\downarrow 10,4$ & $\downarrow 10,8$ & - & $\uparrow 1,4$ & {$[90]$} \\
\hline DVBD20+50-100 ppm ZnO & $\downarrow 0,12-0,24$ & $\uparrow 6,4$ & $\downarrow 2,2$ & - & $\uparrow 1,7$ & $\uparrow 0,5$ & [92] \\
\hline DUCBD20+50-100 ppm ZnO & $\uparrow 0,12-0,36$ & $\uparrow 4,8-5,9$ & $\uparrow 6-10,8$ & $\uparrow 4,4-11,1$ & - & $\uparrow 2,9-4,7$ & [93] \\
\hline DANBD20+50-100 ppm ZnO & $\uparrow 0,12-0,36$ & $\uparrow 2,6-7,9$ & $\uparrow 1,9-5,7$ & $\uparrow 3,4-6,9$ & $\uparrow 5,5$ & $\uparrow 0,5-1,1$ & [94] \\
\hline DKABD20+50-100 ppm ZnO & $\uparrow 0,12-0,35$ & $\uparrow 2,7-8,1$ & $\uparrow 2,1-4,2$ & - & $\uparrow 1,8$ & $\uparrow 1-1,5$ & [95] \\
\hline DTBD60+8-12 $\mu \mathrm{mol} / \mathrm{L} \mathrm{MnO}_{2}$ & - & $\downarrow 9,4-18,8$ & $\downarrow 7,9-8,5$ & - & - & - & [97] \\
\hline DTBD60+8-12 $\mu \mathrm{mol} / \mathrm{L} \mathrm{NiO}$ & - & $\downarrow 7,5-9,4$ & $\downarrow 3,4-10,2$ & - & - & - & [97] \\
\hline DPIRBD20+50-100 ppm CeO ${ }_{2}$ & $\uparrow 0,2 \downarrow 0,2$ & $\downarrow 7-9,9$ & $\downarrow 10,2 \uparrow 15,3$ & - & - & $\uparrow 1,2-0,7$ & [100] \\
\hline DPAMBDX+0,04-0,08 g/L CeO ${ }_{2}$ & - & $\downarrow 6,9-12,9$ & $\downarrow 10,6-16,9$ & - & - & $\uparrow 0,3-1,1$ & [101] \\
\hline
\end{tabular}


Tablo 1. (Devam1).

\begin{tabular}{|l|c|c|c|c|c|c|c|}
\hline DUCBD20+50-100 $\mathrm{ppm} \mathrm{CeO}_{2}$ & $\uparrow 0,3-0,6$ & $\uparrow 0,03-0,09$ & $\uparrow 2,6$ & $\uparrow 4,4$ & - & $\uparrow 3,7-5,2$ & {$[103]$} \\
\hline DNBD20+50 ppm CeO & $\uparrow 0,2$ & $\downarrow 0,8$ & $\uparrow 1,5$ & - & $\uparrow 0,4$ & $\uparrow 0,09$ & {$[104]$} \\
\hline DJBDX+2,5-15 ppm Ce- $\mathrm{Zr}-\mathrm{O}_{2}$ & $\uparrow 0,58$ & $\uparrow 1,45$ & $\uparrow 2,7-5,6$ & - & - & - & {$[105]$} \\
\hline DPONBD20+100 ppm CaCO & $\uparrow 9,5$ & $\uparrow 9$ & - & $\uparrow 29,7$ & - & $\downarrow 4,2$ & {$[106]$} \\
\hline DPONBD20+100 ppm $\mathrm{TiO}_{2}$ & $\uparrow 12$ & $\uparrow 8,5$ & - & $\uparrow 25$ & - & $\downarrow 0,4$ & {$[106]$} \\
\hline DPALBDX+\%0,1-0,2 $\mathrm{TiO}_{2}$ & - & $\uparrow 0,9-7,1$ & $\begin{array}{c}\uparrow 4,3-15,1 \\
\downarrow 1,2-8,8\end{array}$ & - & $\begin{array}{c}\uparrow 0,2-2,5 \\
\downarrow 0,6-1,1\end{array}$ & $\begin{array}{c}\uparrow 1,4-18,9 \\
\downarrow 1-12,8\end{array}$ & {$[107]$} \\
\hline $\begin{array}{l}\text { DPJBD20+50-100 ppm CeO } \\
\text { ÇTKNT bileșimi }\end{array}$ & $\uparrow 0,001$ & $\rightarrow$ & $\uparrow 3-4,5$ & - & $\downarrow 1,6 \uparrow 0,2$ & - & {$[110]$} \\
\hline DPAMBD10E4S1+100 mg/L n-Al & $\downarrow 0,12$ & $\downarrow 9,4$ & $\uparrow 2$ & $\rightarrow$ & - & $\downarrow 1,4$ & {$[112]$} \\
\hline DSBD10E4S1+100 mg/L Al $\mathrm{O}_{3}$ & $\downarrow 0,8$ & $\downarrow 8,9$ & - & - & $\uparrow 23,8$ & $\downarrow 1$ & {$[113]$} \\
\hline
\end{tabular}

\subsection{Nano materyallerin dizel yakıtı ve emülsiyonlarının özelliklerine etkileri}

Havacılık türbin yakıtına (kerosin) \%0,1-1 oranında $\mathrm{Al}_{2} \mathrm{O}_{3}$ katılmasının viskozite, 1sıl iletkenlik ve 1sı transferini artırdığını, ancak 1sıl değer (özgül 1sı) üzerinde önemli bir etkisinin olmadığı belirlenmiştir [13]. Dizel yakıtına katılan n-Al parçacıklarının yoğunluğu azalttığı, alevlenme (parlama) noktası sıcaklığını artırdığı bunun yakıtın depolama ve nakliye güvenliği için önemli olduğu ve tüm karışım oranlarında kinematik viskozitenin artan sıcaklıkla azaldığı bunun ise yağlama, yakıt atomizasyonu, motor performansı, $\mathrm{HC}$ emisyonu ve is oluşumu açısından önemli olduğunu bildirilmiştir [15]. Dizel yakıtına katılan $\mathrm{Al}_{2} \mathrm{O}_{3}[22,23]$ ile $\mathrm{Fe}_{2} \mathrm{O}_{3}$ ve $\mathrm{Fe}_{3} \mathrm{O}_{4}[27,29]$ oranı arttıkça yoğunluk, viskozite, alevlenme ve tutuşma sıcaklığı ile 1sıl değerin arttığ çeşitli araştırmacılar tarafindan belirlenmiştir. Ancak, $\mathrm{Fe}_{2} \mathrm{O}_{3}$ katkısının alevlenme ve tutuşma sicaklığını düşürdüğü [27] ve $\mathrm{Al}_{2} \mathrm{O}_{3}$ katkısının yakıt özellikleri üzerinde önemli bir etkiye sahip olmadığını gösteren $[30,32]$ hatta alevlenme sıcaklığı ve setan sayısının $\mathrm{Al}_{2} \mathrm{O}_{3}$ katkısıyla azaldığını [33] bildiren çalışma sonuçları da vardır. Grafit oksit (GO) katkısının yoğunluğu bir miktar artırmasına rağmen viskozite, alevlenme sicaklığı ve setan sayısı değerlerini düşürdüğü belirlenmiştir [33]. CuO katkısının ise viskozite, alevlenme ve tutuşma sicaklığını düşürdüğü [34] belirlenmiştir. $\mathrm{MnO}$ ve $\mathrm{MnO}_{2}$ katkısının da viskozite, alevlenme ve tutuşma sicaklığı ile akma ve bulutlanma noktası sıcaklığı değerlerini düşürdüğü [34, 35] setan sayısını ise artırdığı [36] bildirilmiştir. Setan sayısının artması tutuşma karakteristiklerini iyileştirerek tutuşma gecikmesi süresini kısaltmakta ve yanma verimini dolayısıyla motor performansını artırmaktadır. $\mathrm{MgO}$ katkısının viskozite, alevlenme sıcaklığı ile akma ve bulutlanma noktası sicaklıklarında azalmaya neden olduğu [35] CaO katkısının yakıt özelliklerine pek etkisinin olmadığı [36] belirlenmiştir. Akma ve bulutlanma noktası sicaklığının azalması özellikle soğuk havalarda motorun ilk harekete geçişini kolaylaştırması bakımından önemlidir. $\mathrm{CeO}_{2}$ katkısının yoğunluk, viskozite, 1sıl değer, alevlenme ve tutuşma sıcaklığı değerlerini artırdığı birçok çalışmada belirlenmiş [37, 39, 44, 45], $\mathrm{TiO}_{2}$ katkısının ise yoğunluk, viskozite ve tutuşma sıcaklığı değerlerinde az miktarda artış sağladığı bildirilmiştir [46]. Dizel yakıtında çok tabakalı karbon nano tüpler (ÇTKNT) kullanılmasının ise yoğunluk, tutuşma sıcaklığı, 1sıl değer ve setan sayısı değerlerinde artış sağladığı belirlenmiştir [47]. Nano alüminyum (n-Al) ve nano silikon (n-Si) parçacıklarının farklı oranlarda su içeren dizel emülsiyon yakıtlara katılmasının yoğunluk, viskozite ve setan sayısını artırırken 1sıl değeri düşürdüğü bildirilmiştir [49]. Dizel-su emülsiyon yakıtlarına ÇTKNT katılmasının yoğunluk, viskozite, alevlenme ve tutuşma sıcaklığı ile setan sayısında artışa ısıl değer de ise düşüşe neden olduğu belirlenmiştir [50, 51]. Dizel-etanol karışımlarında viskozite ve alevlenme sıcaklığının etanol katkısı nedeniyle önemli oranda azaldığı $\mathrm{CeO}_{2}$ katkısının ise yakıt özellikleri üzerinde çok az etkisinin olduğu belirlenmiştir [54]. 


\subsection{Nano materyallerin biyodizel yakıtları ve emülsiyonlarının özelliklerine etkileri}

Farklı türden biyodizel yakıtlara $\mathrm{n}-\mathrm{Al}$, $\mathrm{n}-\mathrm{Ag}$ ve nano grafit $(\mathrm{n}-\mathrm{G})$ parçacıkları katılmasının yoğunluk ve viskoziteyi artırırken alevlenme sıcaklığı ve isıl değerde düşüşe neden olduğu tespit edilmiştir [58, 59, 60, 62]. Pamuk yağı biyodizel yakıtına n-Mn parçacıkları katılmasının yoğunluk, viskozite ve tutuşma sıcaklığını düşürürken 1sıl değerde bir miktar artış sağladığı belirlenmiştir [61]. Farklı türden biyodizel yakıtlara $\mathrm{Al}_{2} \mathrm{O}_{3}, \mathrm{Fe}_{3} \mathrm{O}_{4}$ ve $\mathrm{CeO}_{2}$ katılmasının tüm yakıt özelliklerini artırdığı yönünde çalışma sonuçları $[63,64]$ olmakla birlikte bu katkıların özellikle tutuşma sıcaklığı ve 1s1l değerde düşüşe neden olduğu yönünde [62] bulgular da mevcuttur. Kannan vd. [66] atık kızartma yağı biyodizel yakıtına farklı oranlarda $\mathrm{FeCl}_{3}$ katkısının yoğunluk, viskozite, alevlenme ve tutuşma sıcaklığını düşürürken setan sayısı ve 1sıl değerde artış sağladığını ancak akma ve bulutlanma noktası sıcaklıkları üzerinde bir etkisinin olmadığını belirlemiştir. Sajith vd. [69] Jatropha biyodizel yakıtına katılan $\mathrm{CeO}_{2}$ katkısının alevlenme noktası sicaklığını artırdığını ve viskozitenin artan sicaklıkla azaldığını ancak $\mathrm{CeO}_{2}$ katkısının bulutlanma ve akma noktası sıcaklıkları üzerinde bir etkisinin olmadığını belirlemişlerdir. Karbon nano tüp (KNT) katkıların farklı türde biyozel yakıtlara katılmasının genelde yoğunluk, viskozite, alevlenme sıcaklığı ve setan sayısında artış sağlarken ısıl değerde düşüşe neden olduğu bildirilmektedir $[71,72,73]$. Su içeren biyodizel emülsiyon yakıtlara $\mathrm{CeO}_{2}$ ve $\mathrm{KNT}$ katılmasının yoğunluk, viskozite, setan sayısı ve 1sıl değerde artış sağlarken alevlenme noktası sıcaklığını düşürdüğü belirlenmiştir [76, 77].

\subsection{Nano materyallerin dizel-biyodizel karışımlarının özelliklerine etkileri}

Dizel-palmiye yağı biyodizel karışımlarına nano biyo-polimer parçacıkları katılmasının yoğunluk ve viskozitede düşüş sağlarken 1sıl değeri artırdığı ancak alevlenme sıcaklığı üzerinde etkisinin olmadığı bildirilmiştir [79]. Farklı türden biyodizel-dizel karışımlarına $\mathrm{Al}_{2} \mathrm{O}_{3}$ katılmasının yoğunluk, viskozite, alevlenme sıcaklığ1 ve 1sıl değerde artış sağladığ belirlenmiş [82, 83, 84, 85] olmakla birlikte isıl değeri düşürdüğü yönünde bulgularda vardır [86]. Dizel-biyodizel karışımlarına $\mathrm{CuO}$ katılmasının 1sıl değer dışındaki yakıt özelliklerinde düşüş sağladığı bildirilmiştir [90, 91]. Farklı türden biyodizel-dizel karışımlarına $\mathrm{ZnO}$ katılmasının tüm yakıt özelliklerinde artış sağladığını bildiren çalışmalar [92, 94, 95] olmasına rağmen yoğunluk ve tutuma sıcaklığını azalttığı yönünde bulgular da vardır [92]. Dizel-biyodizel karışımlarına $\mathrm{Fe}_{3} \mathrm{O}_{4}$ katılmasının yoğunluk ve viskozitede artış isıl değer de ise düşüş sağladığı bildirilmiştir [96]. Farklı türden biyodizel-dizel karışımlarına $\mathrm{MnO}_{2}$ ve $\mathrm{NiO}$ katkısının viskozite ve alevlenme sıcaklığını düşürdüğü belirlenmiştir [97]. Dizel-biyodizel karışımlarına $\mathrm{CeO}_{2}$ katılmasının yoğunluk ve tutuşma sıcaklığını düşürdüğü yönünde çalışma sonuçları $[100,101]$ olmakla birlikte tüm yakıt özelliklerinde artış sağladığını bildiren çalışmalar [103, 104] da vardır. Faklı oranlarda biyodizel içeren yakıt karışımlarına $\mathrm{Ce}-\mathrm{Zr}-\mathrm{O}_{2}$ katılmasının yoğunluk, viskozite ve alevlenme sıcaklığını artırdığı bildirilmiştir [105]. Dizel-biyodizel karışımlarına $\mathrm{TiO}_{2}$ katılmasının bazı yakıt özelliklerinde artış sağlarken bazılarında ise düşüş sağladığ ÇTKNT katılmasının setan sayısını düşürürken tutuşma sıcaklığında artış sağladığı belirlenmiştir [110]. Etanol içeren dizel-biyodizel karışımlarına $\mathrm{n}-\mathrm{Al}$ ve $\mathrm{Al}_{2} \mathrm{O}_{3}$ katılması yoğunluk, viskozite ve 1sıl değerde azalmaya neden olduğu $[112,113]$, n-Al katkısının tutuşma sıcaklığını [112] ve $\mathrm{Al}_{2} \mathrm{O}_{3}$ katkısının ise setan sayısını artırdığı [113] belirlenmiştir. 


\section{Nano materyal içerikli katkıların performans karakteristiklerine etkileri}

Nano materyal içerikli katkıların motor performansına etkilerine yönelik sayısal değerler Tablo 2'de verilmiştir. Dizel yakıtına katılan n-Al'nin yanma sonunda oluşan suyun oksijeni ile reaksiyona girerek hidrojenin serbest kalmasını sağlayıp yanma esnasında daha fazla enerji açığa çıkması sonucunda efektif verimi artırıp özgül yakıt tüketimini düşürdüğü belirtilmiştir. Ayrıca, n-Al'nin tutuşma gecikmesi süresini kısaltması, 1sıl değerinin yüksek olması ve katalizör etkisi sayesinde yanma verimini artırarak efektif verimin artmasına dolayısıyla özgül yakıt tüketiminin azalmasına katkı sağladığı bildirilmiştir. Diğer taraftan, n-Al'nin oluşturduğu mikro patlamaların silindir basınç ve sıcaklığını artırmasıyla tam yanma oluşumu sayesinde efektif verimde artış, özgül yakıt tüketiminde ise düşüş olduğu bildirilmiştir [14-16]. Dizel yakıtına katılan n-Ag'nin yakıt demeti nüfuz derinliğini artırıp yakıtla havanın daha iyi karışmasını sağladığı ve tutuşma gecikmesi süresini kısaltıp yanma hızını artırdığı böylece efektif güçte artış, özgül yakıt tüketiminde düşüş sağladığı belirtilmiştir [17]. Mehta vd [20] dizel yakıtına $n-F e, n-A l$ ve $n-B$ katılmasının tutuşma gecikmesi süresini kısalması ve 1sıl değeri artırması sonucunda yanmayı iyileştirerek özgül yakıt tüketiminde düşüş, efektif verimde ise artış sağladığını bildirmişlerdir. Dizel yakıtına katılan $\mathrm{Fe}_{2} \mathrm{O}_{3}$ ve $\mathrm{Fe}_{3} \mathrm{O}_{4}$ 'nin setan sayısı ve 1sıl değer gibi yakıt özelliklerini olumlu yönde etkilemesi ve tutuşma gecikmesi süresini kısaltmasının yanma verimi artırıp özgül yakıt tüketiminde düşüş, efektif verimde ise artış sağladığı bildirilmektedir. Ayrıca, $\mathrm{Fe}_{2} \mathrm{O}_{3}$ ve $\mathrm{Fe}_{3} \mathrm{O}_{4}$ katkısının yakıt demeti nüfuz derinliğini artırarak yanma verimini artırdığı ve yüksek yüzey alanı/hacim oranı sayesinde artan kimyasal aktivitesinin de yanma veriminin artışına katkı sağladığı belirtilmiştir. Diğer taraftan, $\mathrm{Fe}_{2} \mathrm{O}_{3}$ ve $\mathrm{Fe}_{3} \mathrm{O}_{4}$ 'nin yapısında bulunan oksijenin yanmaya olumlu katkı yaptığı da belirtilmektedir. Ancak, nano materyallerin yüksek viskozitesinin püskürtme ve dolayısıyla yanma karakteristiklerini olumsuz yönde etkileyerek özgül yakıt tüketimini artırdığı da tespit edilmiştir [25-29]. Dizel yakıtına katılan $\mathrm{Al}_{2} \mathrm{O}_{3}$ 'nin yakıtın buharlaşma süresini kısalması ile tutuşma gecikmesi süresinin kısaldığı bunun sonucunda yanma veriminin artmasıyla efektif verimin arttığ atomizasyon sağlamasıyla efektif verimde artış, özgül yakıt tüketiminde ise düşüş olduğu bildirilmiştir. $\mathrm{Al}_{2} \mathrm{O}_{3}$ 'nin yakıtın 1 sıl değerinin artırması ve katalizör etkisi sayesinde yanma verimini artırarak efektif verimin artmasına ve özgül yakıt tüketiminin azalmasına katkı sağladığı bildirilmiştir. Ayrıca, $\mathrm{Al}_{2} \mathrm{O}_{3}$ 'nin yanma odasında su buharıyla reaksiyona girmesi sonucu hidrojenin serbest kalmasiyla yanma esnasinda daha fazla enerji açığa çıktığı buna bağlı olarak efektif verimin arttığı ifade edilmektedir. Diğer taraftan, $\mathrm{Al}_{2} \mathrm{O}_{3}$ 'nin yanma odasındaki karbon birikintilerini yakmas1 sonucunda yanma verimini artırdığı ve özgül yakıt tüketimini azalttığı belirtilmektedir. $\mathrm{Al}_{2} \mathrm{O}_{3}$ 'nin yapısında bulunan oksijenin yanmaya olumlu katkı yaptığı belirtilmektedir. Ancak, $\mathrm{Al}_{2} \mathrm{O}_{3}$ 'nin yüksek viskozitesinin püskürtme ve dolayısıyla yanma karakteristiklerini olumsuz yönde etkileyip özgül yakıt tüketimini artırdığı yönünde yorumlar da mevcuttur [21-23, 30]. George $v d$ [31] dizel yakıtına katılan $\mathrm{Al}_{2} \mathrm{O}_{3}$ ve $\mathrm{Co}_{3} \mathrm{O}_{4}$ 'nin yanma karakteristiklerini iyileştirerek yakıtın daha iyi yakılması sonucu efektif verimde artış, özgül yakıt tüketiminde ise düşüş sağladığını bildirmişlerdir. Dizel yakıtına katılan $\mathrm{CuO}$ 'in yapısında bulunan oksijen sayesinde yakıtın oksidasyon oranını artırması, setan sayısı ve 1sıl değer gibi yakıt özelliklerini iyileştirmesi ve tutuşma gecikmesi süresini azaltması sonucunda efektif güçte ve efektif verimde artış, özgül yakıt tüketiminde ise düşüş sağladığı belirtilmektedir [30-34]. Dizel yakıtına katılan MnO'in katalizör etkisi ve tutuşma gecikmesi süresini kısaltması sonucunda efektif 
verimde artış, özgül yakıt tüketiminde ise düşüş sağladığ 1 tespit edilmiştir [34, 35]. Dizel yakıtına katılan MgO'in katalizör etkisi sayesinde yanmayı iyileştirerek özgül yakıt tüketiminde azalma sağladı̆̆ yakıtına katılan ZnO'in yanma sırasında açığa çıkan ısı miktarını artırarak efektif verimi de artırdığını belirlemişlerdir. Dizel yakıtına katılan $\mathrm{CeO}_{2}$ 'in sağladığı ekstra oksijen ve katalizör etkisi sayesinde yanma verimi artırarak efektif verimde iyileşme sağladığ1 belirlenmiştir [37]. $\mathrm{CeO}_{2}$ 'in yanmayı iyileştirmesi sonucunda özgül yakıt tüketiminin azaldığ 1 ve efektif verimi artırdığ $[39,40,42,44,45]$, bunun yanında yakıtın isıl değerini düşürmesi nedeniyle efektif verimi azalttığı $[41,43]$ yönünde farklı bulgular da bulunmaktadır. Fangsuwannarak vd [46] dizel yakıtına $\mathrm{TiO}_{2}$ katılmasının efektif güç ve momentte artış özgül yakıt tüketiminde ise azalma sağladığını bildirmişlerdir. Aalam vd [47] dizel yakıtına katılan ÇKKNT'in yakıtın fiziksel özelliklerini iyileştirmesi sonucunda püskürtülen yakıtın daha iyi atomize olmasını ve yanma odasında yakıtın havayla daha iyi karışmasını sağlayarak yanma verimini artırıp efektif verimde artış, özgül yakıt tüketiminde ise düşüş elde edildiğini bildirmişlerdir.

Tablo 2. Nanomateryal içerikli katkıların performans karakteristiklerine etkileri.

\begin{tabular}{|c|c|c|c|c|c|}
\hline Ana yakıt+katk1 & $\begin{array}{l}\text { Efektif güç } \\
\text { (\%değişim) }\end{array}$ & $\begin{array}{c}\text { Moment } \\
\text { (\%değişim) }\end{array}$ & $\begin{array}{l}\text { Efektif verim } \\
\text { (\%değişim) }\end{array}$ & $\begin{array}{l}\text { Özgül yakıt } \\
\text { tüketimi } \\
\text { (\%değişim) }\end{array}$ & Kaynak \\
\hline $\mathrm{D}+30-50 \mathrm{cc} / \mathrm{L} \mathrm{n}-\mathrm{Al}$ & - & - & - & $\downarrow$ & [14] \\
\hline $\mathrm{D}+25-75 \mathrm{ppm} \mathrm{n-Al}$ & - & - & $\uparrow$ & $\downarrow$ & [15] \\
\hline $\mathrm{D}+\% 0,5 \mathrm{n}-\mathrm{Al}$ & - & - & $\uparrow 9$ & $\downarrow 7$ & [16] \\
\hline $\mathrm{D}+10-40 \mathrm{ppm} n-\mathrm{Ag}$ & $\uparrow 1,1-7,3$ & - & - & $\downarrow 1-2$ & [17] \\
\hline $\mathrm{D}+\mathrm{n}-\mathrm{Fe}, \mathrm{n}-\mathrm{Al}, \mathrm{n}-\mathrm{B}$ & - & - & $\uparrow 2-9$ & $\uparrow 7$ & [20] \\
\hline $\mathrm{D}+\% 0,4-0,8 \mathrm{Fe}_{3} \mathrm{O}_{4}$ & - & - & $\uparrow 3,3-12,2$ & $\downarrow 3,2-10,9$ & [25] \\
\hline $\mathrm{D}+\% 0,4-0,8 \mathrm{Fe}_{3} \mathrm{O}_{4}$ & - & - & - & $\downarrow 34$ & [26] \\
\hline $\mathrm{D}+150-300 \mathrm{mg} / \mathrm{L} \mathrm{Fe}_{3} \mathrm{O}_{4}$ & - & - & $\uparrow 23-36$ & $\downarrow 26$ & [27] \\
\hline $\mathrm{D}+\% 4-12 \mathrm{Fe}_{3} \mathrm{O}_{4}$ & - & - & $\uparrow 6,2-21,4$ & $\downarrow 6,1-18,2$ & [28] \\
\hline $\mathrm{D}+25-50 \mathrm{ppm} \mathrm{Fe}_{2} \mathrm{O}_{3}$ ve $\mathrm{Fe}_{3} \mathrm{O}_{4}$ & - & - & $\uparrow 2$ & $\downarrow 9$ & [29] \\
\hline $\mathrm{D}+1-1,5 \mathrm{~g} / \mathrm{L} \mathrm{Al}_{2} \mathrm{O}_{3}$ & - & - & $\uparrow 2,3-6,2$ & - & [21] \\
\hline $\mathrm{D}+25-50 \mathrm{ppm} \mathrm{Al}_{2} \mathrm{O}_{3}$ & - & - & $\uparrow 3-6$ & $\downarrow 4-7$ & [22] \\
\hline $\mathrm{D}+250-1000 \mathrm{ppm} \mathrm{Al}_{2} \mathrm{O}_{3}$ & - & - & $\uparrow 6$ & - & [23] \\
\hline $\mathrm{D}+25-100 \mathrm{ppm} \mathrm{Al}_{2} \mathrm{O}_{3}$ & $\uparrow 3,3$ & $\uparrow 3,3$ & - & $\downarrow 1,2$ & {$[30]$} \\
\hline $\mathrm{D}+50 \mathrm{ppm} \mathrm{Al}_{2} \mathrm{O}_{3}$ ve $\mathrm{Co}_{3} \mathrm{O}_{4}$ & - & - & $\uparrow$ & $\downarrow$ & [31] \\
\hline $\mathrm{D}+50 \mathrm{ppm} \mathrm{CuO}$ & $\uparrow_{1}$ & $\uparrow 1$ & - & $\downarrow 0,5$ & [30] \\
\hline $\mathrm{D}+200 \mathrm{mg} / \mathrm{L} \mathrm{CuO}$ & - & - & $\downarrow$ & - & [34] \\
\hline $\mathrm{D}+200 \mathrm{mg} / \mathrm{L} \mathrm{MnO}$ & - & - & $\uparrow 4$ & - & [34] \\
\hline $\mathrm{D}+8-16 \mu \mathrm{mol} / \mathrm{L} \mathrm{MnO}$ & $\uparrow$ & $\uparrow$ & - & $\downarrow 2,2-4,2$ & [35] \\
\hline $\mathrm{D}+8-16 \mu \mathrm{mol} / \mathrm{L} \mathrm{MgO}$ & - & - & - & $\downarrow 1-2$ & [35] \\
\hline $\mathrm{D}+250-500 \mathrm{ppm} \mathrm{ZnO}$ & - & - & $\uparrow 1-1,5$ & - & [24] \\
\hline $\mathrm{D}+50 \mathrm{cc} / \mathrm{L} \mathrm{CeO}{ }_{2}$ & - & - & $\uparrow 6$ & - & [37] \\
\hline $\mathrm{D}+50 \mathrm{cc} / \mathrm{L} \mathrm{CeO}_{2}$ & - & - & - & $\downarrow 6,3$ & [39] \\
\hline $\mathrm{D}+0,05-5 \mathrm{~mL} / \mathrm{L} \mathrm{CeO}_{2}$ & - & - & - & $\downarrow 11,3$ & [40] \\
\hline $\mathrm{D}+25 \mathrm{ppm} \mathrm{CeO}_{2}$ & - & - & $\downarrow$ & - & [41] \\
\hline $\mathrm{D}+25-50 \mathrm{ppm} \mathrm{CeO}{ }_{2}$ & - & - & $\uparrow 2,4-5,3$ & $\downarrow 9$ & [42] \\
\hline $\mathrm{D}+10-40 \mathrm{ppm} \mathrm{CeO}{ }_{2}$ & - & - & - & $\downarrow$ & [43] \\
\hline $\mathrm{D}+25-50 \mathrm{ppm} \mathrm{CeO}_{2}$ & - & - & $\uparrow 6$ & - & [44] \\
\hline $\mathrm{D}+50 \mathrm{cc} / \mathrm{L} \mathrm{CeO}{ }_{2}$ & - & - & $\uparrow 5,8$ & $\downarrow 6,3$ & [45] \\
\hline \begin{tabular}{|l}
$\mathrm{D}+\% 0,2 \mathrm{TiO}_{2}$ \\
\end{tabular} & $\uparrow$ & $\uparrow 1-2,3$ & - & $\downarrow 13,6$ & [46] \\
\hline $\mathrm{D}+25-50$ ppm ÇTKNT & - & - & $\uparrow 2,5$ & $\downarrow 0,3-0,5$ & {$[47]$} \\
\hline DW15S2+25-100 ppm n-Al & - & - & $\uparrow 1,7-3,7$ & $\downarrow 3$ & [48] \\
\hline DW1+\%0,1 n-Al & - & - & $\uparrow 16$ & $\downarrow 21$ & [49] \\
\hline DW1+\%0,1 n-Si & - & - & $\uparrow 14$ & $\downarrow 37$ & [49] \\
\hline DW15S2+50-150 ppm ÇTKNT & - & - & $\uparrow 0,3-1,9$ & $\downarrow 10$ & [50] \\
\hline DW20S2+50-150 ppm ÇTKNT & - & - & $\uparrow 0,9-1,6$ & $\downarrow 9,7$ & [51] \\
\hline DW5+\%3 Flyash & - & 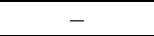 & $\uparrow 0,3-2,4$ & $\uparrow 2,9-4,6$ & [52] \\
\hline $\mathrm{DE} 10+10-20 \mathrm{~g} / \mathrm{L} \mathrm{CeO}_{2}$ & - & - & $\downarrow$ & $\downarrow 1,2-1,4$ & [54] \\
\hline DE15+50-75 ppm $\mathrm{Al}_{2} \mathrm{O}_{3}$ & - & - & $\uparrow 7$ & $\downarrow 11,1$ & [55] \\
\hline DE15+50-75 $\mathrm{ppm} \mathrm{Fe}_{3} \mathrm{O}_{4}$ & - & - & $\uparrow$ & $\downarrow$ & [55] \\
\hline JBD+50 ppm n-Al & - & - & $\uparrow 3$ & $\downarrow 13,5$ & {$[56]$} \\
\hline
\end{tabular}


Tablo 2. (Devam1).

\begin{tabular}{|c|c|c|c|c|c|}
\hline $\mathrm{JBD}+100 \mathrm{mg} / \mathrm{L} \mathrm{n}-\mathrm{Al}-\mathrm{Mg}$ & - & - & $\uparrow 0,6$ & $\downarrow 3$ & {$[57]$} \\
\hline HBD+50 ppm n-Ag & - & - & $\uparrow$ & - & {$[58]$} \\
\hline $\mathrm{HBD}+25-50 \mathrm{ppm}$ n-Ag & - & - & $\uparrow$ & - & {$[59]$} \\
\hline $\mathrm{HBD}+50 \mathrm{ppm} \mathrm{n-G}$ & - & - & $\uparrow$ & - & {$[58]$} \\
\hline $\mathrm{HBD}+25-50 \mathrm{ppm} \mathrm{n-G}$ & - & - & $\uparrow 0,2-5,8$ & - & {$[60]$} \\
\hline PAMBD+4-16 $\mu \mathrm{mol} / \mathrm{L} \mathrm{n}-\mathrm{Mn}$ & - & $\uparrow 3,5-6$ & $\uparrow 5$ & $\downarrow 5,5$ & [61] \\
\hline $\mathrm{JBD}+30 \mathrm{ppm} / \mathrm{L} \mathrm{Al}_{2} \mathrm{O}_{3}$ & - & - & $\uparrow 1,7$ & $\downarrow 5,3$ & {$[62]$} \\
\hline $\mathrm{NBD}+100-300 \mathrm{ppm} \mathrm{Al}_{2} \mathrm{O}_{3}$ & - & - & $\uparrow 2,2-4,2$ & $\downarrow$ & [63] \\
\hline $\mathrm{ATBD}+5-50 \mu \mathrm{mol} / \mathrm{L} \mathrm{FeCl}_{3}$ & - & - & $\uparrow 3,1$ & $\downarrow 18,4$ & {$[64]$} \\
\hline $\mathrm{JBD}+10-600 \mathrm{ppm} \mathrm{Fe}_{2} \mathrm{O}_{3}$ ve $\mathrm{Co}_{3} \mathrm{O}_{4}$ & - & - & $\uparrow 0,5$ & $\downarrow 3,6$ & [65] \\
\hline $\mathrm{MBD}+\% 1 \mathrm{Fe}_{3} \mathrm{O}_{4}$ & - & - & $\uparrow 5,1$ & $\downarrow 4,7$ & {$[66]$} \\
\hline $\mathrm{JBD}+100 \mathrm{mg} / \mathrm{L} \mathrm{Co}_{3} \mathrm{O}_{4}$ & - & - & $\uparrow 0,4$ & $\downarrow 2$ & {$[57]$} \\
\hline $\mathrm{CALBD}+150 \mathrm{mg} / \mathrm{L} \mathrm{Co}_{3} \mathrm{O}_{4}$ & - & - & $\uparrow 7$ & $\downarrow 4$ & {$[67]$} \\
\hline CALBD+150 mg/L $\mathrm{TiO}_{2}$ & - & - & $\uparrow 5$ & $\downarrow 2$ & [67] \\
\hline $\mathrm{PONBD}+\mathrm{Rh}_{2} \mathrm{O}_{3}$ & - & - & $\uparrow 1$ & $\downarrow 3$ & [68] \\
\hline $\mathrm{SBBD}+25 \mathrm{ppm} / \mathrm{L} \mathrm{CeO}_{2}$ & - & - & $\downarrow$ & - & [41] \\
\hline $\mathrm{JBD}+30 \mathrm{ppm} / \mathrm{L} \mathrm{CeO}_{2}$ & - & - & $\uparrow 1,6$ & $\downarrow 4,7$ & [62] \\
\hline $\mathrm{JBD}+20-80 \mathrm{ppm} \mathrm{CeO}_{2}$ & - & - & $\uparrow 1,5$ & - & [69] \\
\hline $\mathrm{JBD}+25-50 \mathrm{ppm} \mathrm{KNT}$ & - & - & $\uparrow 2,2-4$ & $\downarrow 10,8-16,2$ & {$[56]$} \\
\hline NBD+100-300 ppm KNT & - & - & $\uparrow 2,1-4,2$ & $\downarrow$ & {$[71]$} \\
\hline AYBD+100ppm KNT & - & - & $\uparrow 19,9$ & $\downarrow 25$ & {$[72]$} \\
\hline HBD+50 ppm ÇTKNT & - & - & $\uparrow$ & - & {$[58]$} \\
\hline HBD+25-50 ppm ÇTKNT & - & - & $\uparrow 2$ & - & {$[73]$} \\
\hline JBDW15S2+25-100 ppm n-Al & - & - & $\uparrow 1,6-3,9$ & $\downarrow 8,3-13,9$ & [74] \\
\hline KABDW15S2+50 ppm $\mathrm{CeO}_{2}$ & - & - & $\uparrow$ & $\downarrow$ & [75] \\
\hline LOBDW5S2+30 ppm $\mathrm{CeO}_{2}$ & - & - & $\uparrow$ & $\downarrow$ & {$[76]$} \\
\hline JBDW5S2+25-100 ppm KNT & - & - & $\uparrow 1,6-2,1$ & $\downarrow 9-13$ & {$[77]$} \\
\hline DSBD10+\%1,5 n-Cu & - & - & $\uparrow 1$ & - & {$[12]$} \\
\hline DATBD20+25-75 ppm Al $\mathrm{Al}_{3}$ & - & - & $\uparrow$ & $\downarrow$ & {$[80]$} \\
\hline $\mathrm{DPLBD} 20+30 \mathrm{mg} / \mathrm{L} \mathrm{Al}_{2} \mathrm{O}_{3}$ & - & - & $\uparrow$ & - & [81] \\
\hline DZJBD25+25-50 ppm $\mathrm{Al}_{2} \mathrm{O}_{3}$ & - & - & $\uparrow 2,5$ & $\downarrow 6$ & {$[82]$} \\
\hline DUCBD20+50-100 ppm Al $\mathrm{A}_{3}$ & - & - & $\uparrow$ & $\downarrow$ & {$[83]$} \\
\hline DDBDX+100 ppm $\mathrm{Al}_{2} \mathrm{O}_{3}$ & - & - & $\uparrow 22$ & $\downarrow 17$ & {$[86]$} \\
\hline DJBD20+50-150 ppm $\mathrm{Al}_{2} \mathrm{O}_{3}$ & - & - & $\uparrow$ & $\downarrow$ & {$[87]$} \\
\hline DMBD20+40-80 ppm $\mathrm{Al}_{2} \mathrm{O}_{3}$ & - & - & $\uparrow 0,8-1$ & $\downarrow 5,1-7,7$ & {$[88]$} \\
\hline DMBD20+40-80 ppm Fe $\mathrm{O}_{4}$ & - & - & $\uparrow 0,5-0,6$ & $\downarrow 2,6$ & {$[88]$} \\
\hline DKEBD20+40-120 ppm CuO & - & - & $\uparrow 3-4$ & $\downarrow$ & [89] \\
\hline DMBD20+50 ppm CuO & - & - & $\uparrow 2,2$ & $\downarrow$ & {$[90]$} \\
\hline DMBD20+50 ppm CuO & - & - & $\uparrow$ & - & [91] \\
\hline DMBD20+50 ppm MgO & - & - & $\uparrow$ & - & {$[91]$} \\
\hline DVBD20+50-100 ppm ZnO & - & - & $\uparrow$ & $\downarrow$ & [92] \\
\hline DUCBD20+50-100 ppm ZnO & - & - & $\uparrow$ & $\downarrow$ & [93] \\
\hline DANBD20+50-100 ppm ZnO & - & - & $\uparrow 2,8$ & $\downarrow 3,8$ & [94] \\
\hline DKABD20+50-100 ppm ZnO & - & - & $\uparrow$ & $\downarrow$ & [95] \\
\hline DTBD60+8-12 $\mu \mathrm{mol} / \mathrm{L} \mathrm{MnO}_{2}$ & - & - & - & $\downarrow$ & [97] \\
\hline DTBD60+8-12 $\mu \mathrm{mol} / \mathrm{L} \mathrm{NiO}$ & - & - & - & $\downarrow$ & {$[97]$} \\
\hline $\mathrm{DPONBDX}+\mathrm{Rh}_{2} \mathrm{O}_{3}$ & - & - & $\uparrow$ & $\downarrow 3$ & {$[98]$} \\
\hline DPALBDX+3 mg/L CeO ${ }_{2}$ & - & - & $\downarrow$ & $\uparrow$ & [99] \\
\hline DPIRBD20+50-100 ppm $\mathrm{CeO}_{2}$ & - & - & $\uparrow$ & $\downarrow$ & {$[100]$} \\
\hline DPAMBDX+0,04-0,08 g/L CeO ${ }_{2}$ & - & - & $\uparrow$ & $\downarrow$ & {$[101]$} \\
\hline DUCBD20+50-100 ppm CeO $\mathrm{Ce}_{2}$ & - & - & $\uparrow$ & $\downarrow$ & {$[103]$} \\
\hline DNBD20+50 ppm $\mathrm{CeO}_{2}$ & - & - & $\uparrow 2,8$ & $\uparrow$ & {$[104]$} \\
\hline DJBDX+2,5-15 ppm Ce-Zr-O ${ }_{2}$ & - & - & $\uparrow 8,7-15,3$ & - & {$[105]$} \\
\hline DPONBD20+100 ppm $\mathrm{TiO}_{2}$ & - & - & $\uparrow 2,1$ & $\downarrow 5,7$ & [106] \\
\hline DPALBDX+\%0,1-0,2 $\mathrm{TiO}_{2}$ & $\uparrow 1,6-2,4$ & $\uparrow$ & - & $\downarrow$ & {$[107]$} \\
\hline DNBD20+250-500 ppm $\mathrm{TiO}_{2}$ & - & - & $\uparrow 8,9-9,6$ & $\downarrow 3,4-5,5$ & [108] \\
\hline DATBDX+30-90 ppm $\mathrm{CeO}_{2}$ ve ÇTKNT & $\uparrow 0,6-7,8$ & $\uparrow 1,2-4,9$ & - & $\downarrow 0,3-4,5$ & [109] \\
\hline DATBD20+30 ppm ÇTKNT & $\uparrow 17$ & $\uparrow 18$ & - & $\downarrow$ & [111] \\
\hline DPAMBD10E4S1+100 mg/L n-Al & - & - & $\uparrow$ & $\downarrow$ & {$[112]$} \\
\hline DSBD10E4S1+100 mg/L Al $\mathrm{O}_{3}$ & - & - & $\uparrow$ & $\downarrow$ & [113] \\
\hline DBD10E20+25-100 ppm $\mathrm{CeO}_{2}$ ve $\mathrm{KNT}$ & - & - & $\uparrow 1,9$ & $\downarrow 7,7$ & {$[114]$} \\
\hline
\end{tabular}

\%15 su içeren dizel emülsiyon yakıtına katılan n-Al’nin meydana getirdiği mikro patlamalar sonucu oluşan ikincil atomizasyon ve yüksek alan/hacim oranı sayesinde 
yakıtın daha verimli yanmasıyla efektif verimde artış, özgül yakıt tüketiminde ise düşüş olduğunu belirlenmiştir [48]. \%1 su içeren dizel emülsiyon yakıtına katılan n-Al ve nSilikon'un su ile reaksiyona girmesi sonucu serbest kalan hidrojenin yanma sirasinda açığa çıkan enerjiyi artırarak özgül yakıt tüketiminin azalmasını ve efektif verimin artmasını sağladığı belirtilmektedir. Ayrıca, n-Al ve n-Silikon'un su ile reaksiyonunun egzotermik bir reaksiyon olması sebebiyle yanma odasında fazladan 1s1 oluşumu sayesinde performans parametrelerinin iyileşmesine katkı sağladığı da ifade edilmiştir [49]. \%15 ve 20 su içeren dizel emülsiyon yakıtlarına katılan ÇTKNT katkısının yüksek buharlaşma oranı sayesinde tutuşma gecikmesi süresini kısaltarak daha fazla miktarda yakıtın yakılabilmesini ve bunun sonucunda efektif verimin artmasına ve özgül yakıt tüketiminin azalmasına katkı sağladığı belirtilmiştir. Ayrıca, nano materyal maddelerin yüksek alan/hacim oranı sayesinde yanma sirasında daha fazla kimyasal aktivite gösterip yanma verimini artırarak motor performansını ve yakıt tüketimini iyileştirdiği ifade edilmiştir [50, 51]. \%5 ve 10 su içeren dizel emülsiyon yakıtlarına katılan Flyash katkısının özgül yakıt tüketimini az miktarda artırdığı, efektif verimi ise az miktarda düşürdügü bildirilmiştir [52]. \%10 etanol içeren dizel karışım yakıtına katılan $\mathrm{CeO}_{2}$ katkısının yapısında bulunan oksijen sayesinde yanmayı iyileştirerek efektif verimi artırdığını ancak etanolün ısıl değeri düşürmesi sonucunda özgül yakıt tüketiminin arttığ $\mathrm{Fe}_{3} \mathrm{O}_{4}$ katkılarının efektif verimde artış, özgül yakıt tüketiminde düşüş sağladığı tespit edilmiştir [55]. Farklı türden biyodizel yakıtlara katılan n-Al, n-Mg, n-Mn, n-Ag ve n$\mathrm{G}$ katkılarının genel olarak efektif verimde artış, özgül yakıt tüketiminde ise düşüş sağladığı bildirilmiştir [56-61]. Farklı türden biyodizel yakıtlara katılan $\mathrm{Al}_{2} \mathrm{O}_{3}$ 'in yüksek yüzey alanı/hacim oranı sayesinde püskürtme sırasında yakıtın atomizasyonunu iyileştirip yakıtın hava ile daha iyi karışmasını ve tutuşma gecikmesi süresinin kısalmasını sağlayarak yanma verimini artırdığı bunun sonucunda efektif verimde artış, özgül yakıt tüketiminde ise düşüş meydana geldiği belirtilmiştir [62, 63]. Atık yağ biyodizel yakıtına katılan $\mathrm{FeCl}_{3}$ 'ün katalizör etkisi sayesinde yanma verimini artırarak efektif verimde ve özgül yakıt tüketiminde iyileşme sağladığı tespit edilmiştir [64]. Jatropha yağı biyodizel yakıtına katılan $\mathrm{Fe}_{2} \mathrm{O}_{3}$ ve $\mathrm{Co}_{3} \mathrm{O}_{4}$ 'in efektif verimde ve özgül yakıt tüketiminde iyileşme sağladığı belirlenmiştir [65]. Mustard yağı biyodizel yakıtına katılan $\mathrm{Fe}_{3} \mathrm{O}_{4}$ 'in yanma sırasında daha fazla 1S1 açığa çıkmasını sağlayarak 1s1 yayılım oranını artırdığı ve yapısında bulunan oksijen sayesinde yanmayı iyileştirerek efektif verimde ve özgül yakıt tüketiminde iyileşme sağladığı bildirilmiştir [66]. Farklı türden biyodizel yakıtlara katılan $\mathrm{Co}_{3} \mathrm{O}_{4}$ 'in katalizör etkisi ve yapısında oksijen bulunması sayesinde daha fazla yakıtın yanmasını sağlayarak efektif verimde ve özgül yakıt tüketiminde iyileşme sağladığı bildirilmiştir [57, 67]. Benzer şekilde, Calophyllum yağı biyodizel yakıtına katılan $\mathrm{TiO}_{2}$ 'in de efektif verimde ve özgül yakıt tüketiminde iyileşme sağladığı belirlenmiştir [67]. Pongamia yağı biyodizel yakıtına katılan $\mathrm{Rh}_{2} \mathrm{O}_{3}$ 'in yüksek buharlaşma oranı sayesinde erken buharlaşarak tutuşma gecikmesi süresini kısalttığı ve yüksek kimyasal aktivitesi sayesine yanma verimini artırarak efektif verimin artmasını, özgül yakıt tüketiminin ise azalmasını sağladığı belirlenmiştir [68]. Farklı türden biyodizel yakıtlara katılan $\mathrm{CeO}_{2}$ 'in efektif verimde artış, özgül yakıt tüketiminde ise düşüş sağladığını bildiren çalışmaların $[62,69]$ yanı sıra $\mathrm{CeO}_{2}{ }^{\prime}$ in efektif verimde düşüşe neden olduğunu bildiren çalışmalar [41] da vardır. Farklı türden biyodizel yakıtlarına katılan KNT ve ÇTKNT katkılarının yanmayı iyileştirici özellikleri sayesinde yakıtın daha fazla yakılabildiği ve bunun sonucunda efektif verimde artış, özgül yakıt tüketiminde ise düşüş elde edildiği bildirilmektedir [56, 58, 71-73]. \%15 su içeren Jatropha yağı biyodizel emülsiyon yakıtına katılan n-Al parçacıklarının meydana getirdiği mikro patlamaların ikincil bir atomizasyon oluşturup 
yanma verimini artırarak efektif verimde artış, özgül yakıt tüketiminde ise düşüş sağladığı belirtilmektedir [74]. \%15 su içeren kanola yağı biyodizel emülsiyon yakıtına katılan $\mathrm{CeO}_{2}$ 'in yapısında bulunan oksijen sayesinde püskürtülen yakıtın daha fazla yakılmasını sağlayarak efektif verimde artış, özgül yakıt tüketiminde ise düşüş sağladığ1 tespit edilmiştir [75]. \%5 su içeren limon otu yağı biyodizel emülsiyon yakıtına katılan $\mathrm{CeO}_{2}$ 'in yüksek yüzey alanı/hacim oranı sayesinde daha hızlı buharlaşması ile tutuşma gecikmesi süresini kısaltmasının ve $\mathrm{CeO}_{2}$ 'in yanma ürünleri içindeki suyun oksijeni ile reaksiyona girerek hidrojeni serbest bırakmasının efektif verimin ve özgül yakıt tüketiminin iyileşmesini sağladığı bildirilmektedir [76]. \%5 su içeren Jatropha yağı biyodizel emülsiyon yakıtına katılan KNT'in meydana getirdiği mikro patlamaların ikincil bir atomizasyon oluşturup yanma hızını artırarak efektif verimde artış, özgül yakıt tüketiminde ise azalma sağladığı tespit edilmiştir [77]. \%10 soya yağı biyodizeldizel yakıt karışımına katılan n-Cu parçacıklarının yüksek kimyasal aktivitesi sayesinde yanmayı iyileştirerek efektif verimde artış sağladığı bildirilmiştir [12]. Farklı tür ve oranlarda biyodizel-dizel yakıt karışımlarına katılan $\mathrm{Al}_{2} \mathrm{O}_{3}$ 'in efektif verimde farklı oranlarda artış ve özgül yakıt tüketiminde düşüş sağladığı birçok çalışmada tespit edilmiştir [80-83, 86-88]. Benzer şekilde, \%20 Mahua yağı biyodizel-dizel yakıt karışımına katılan $\mathrm{Fe}_{3} \mathrm{O}_{4}$ 'in yakıt ve yanma özelliklerini iyileştirip tutuşma gecikmesi süresini kısaltarak özgül yakıt tüketiminde azalma, efektif verimde ise artış sağladığı belirtilmiştir [88]. Farklı tür ve oranlarda biyodizel-dizel yakıt karışımlarına katılan $\mathrm{CuO}^{\prime}$ 'in katalizör etkisi ve iyileşen püskürtme karakteristikleri sayesinde efektif verimde artış ve özgül yakıt tüketiminde düşüş sağladığı birçok çalışmada tespit edilmiştir [8991]. Benzer şekilde, \%20 Mahua yağı biyodizel-dizel yakıt karışımına katılan MgO’in yakıt ve yanma özelliklerini iyileştirerek özgül yakıt tüketiminde azalma, efektif verimde ise artış sağladığı bildirilmiştir [91]. Farklı tür ve oranlarda biyodizel-dizel yakıt karışımlarına katılan ZnO'in iyileşen yakıt özellikleri ve kısalan tutuşma gecikmesi süresi sayesinde daha verimli bir yanma sonucunda efektif verimde artış, özgül yakıt tüketiminde düşüş sağladığı birçok çalışmada tespit edilmiştir [92-95]. \%60 Tall yağı biyodizel-dizel yakıt karışımına katılan $\mathrm{MnO}_{2}$ ve NiO'in özgül yakıt tüketiminde azalma sağladığı bildirilmiştir [97]. \%10-30 Pongamia yağı biyodizeldizel yakıt karışımlarına katılan $\mathrm{Rh}_{2} \mathrm{O}_{3}$ ' in katalizör etkisi sayesinde yakılabilen yakıt miktarını artırarak özgül yakıt tüketiminde azalma, efektif verimde artış sağladığı bildirilmiştir [98]. Farklı tür ve oranlarda biyodizel-dizel yakıt karışımlarına katılan $\mathrm{CeO}_{2}$ 'in efektif verimde artış, özgül yakıt tüketiminde düşüş sağladığı birçok çalışmada belirtilmekle [100, 101, 103, 104] birlikte $\mathrm{CeO}_{2}$ katkısının efektif verim ve özgül yakıt tüketimini olumsuz yönde etkilediğini bildiren çalışmalar da vardır [99]. \%5-15 Jatropha biyodizel-dizel yakıt karışımına katılan $\mathrm{Ce}-\mathrm{Zr}-\mathrm{O}_{2}{ }^{\prime}$ in yapısında oksijen bulunması ve katalizör etkisi sayesinde tam yanma sağlayıp efektif verimi artırdığı bildirilmiştir [105]. Farklı tür ve oranlarda biyodizel-dizel yakıt karışımlarına katılan $\mathrm{TiO}_{2}$ 'in efektif güçte, momentte ve efektif verimde artış, özgül yakıt tüketiminde ise düşüş sağladığı birçok çalışmada belirtilmiştir [106-108]. \%5-20 atık yă̆ biyodizel-dizel yakıt karışımlarına katılan $\mathrm{CeO}_{2}$ ve ÇTKNT'in tam yanma gerçekleşmesini sağlayarak efektif güç, moment, efektif verim ve özgül yakıt tüketimi gibi performans parametrelerinde iyileşme sağladığı belirtilmiştir [109]. Benzer şekilde, $\% 20$ atık yă̆ biyodizel-dizel yakıt karışımına katılan ÇTKNT'in de efektif güç ve döndürme momentinde artış, özgül yakıt tüketiminde ise azalma sağladığı belirtilmiştir [111]. Dizel, faklı türden biyodizel ve etanol yakıt karışımlarına katılan $\mathrm{n}-\mathrm{Al}, \mathrm{Al}_{2} \mathrm{O}_{3}$, $\mathrm{CeO}_{2}$ ve KNT gibi farklı nanomateryal içerikli katkıların efektif verimde artış ve özgül yakıt tüketiminde azalma sağladığı farklı çalışmalarda bildirilmiştir [112-114]. 


\section{Sonuçlar ve öneriler}

Bu çalışma, dizel, biyodizel, su içeren emülsiyon yakıtları ve çeşitli yakıt karışımları gibi dizel motor yakıtlarında nano materyal içerikli katkıların kullanımı üzerine yapılmış çeşitli çalışmaların sonuçlarından derlenmiştir. Yapılan bu derleme sonucunda çeşitli nano materyal içerikli katkıların dizel, biyodizel, emülsiyon yakıtları ve çeşitli yakıt karışımlarının özelliklerini iyileştirmek amacıyla kullanılabileceği görülmüştür. Elde edilen bulguların ışığında aşağıdaki sonuçlar özetlenebilir.

- Nano materyal içerikli katkıların alevlenme noktası sıcaklığını artırarak yakıtın depolanması ve nakledilmesi hususunda avantaj oluşturabileceği söylenebilir.

- Nano materyal içerikli katkıların akma ve bulutlanma noktası sıcaklıklarını düşürerek özellikle soğuk havalarda motorun ilk harekete geçişini kolaylaştıracağı söylenebilir.

- Nano materyal içerikli katkıların artırılmış yüzey alanı/hacim oranı, artırılmış radyasyon/kütle transfer özellikleri ve daha iyi tutuşma özellikleri sayesinde iyi bir katalizör olarak yanmanın iyileşmesine katkı sağlayacağı söylenebilir [115].

- Emülsiyon yakıtlara nano materyal içerikli katkıların katılmasının yanma sırasında mikro patlamalar meydana getirerek yanma odasında ikincil atomizasyon oluşturup yanma verimini dolayısıyla motor performansını artırdığ 1 söylenebilir [116, 117].

- Genel olarak nano materyal içerikli katkıların setan sayısı ve 1sıl değeri iyileştirmektedir. Bunun yanma verimini artırarak tam yanmanın gerçekleşmesine katkı sağlayacağı söylenebilir. Yoğunluk, viskozite ve alevlenme noktası sıcaklığı gibi özelliklerdeki artışların düşük seviyelerde olduğu söylenebilir [118].

- Nano materyal içerikli katkıların tutuşma gecikmesi süresini kısaltarak yanmanın daha erken başlamasına ve yanma hızının ve veriminin artmasına katkı sağladığı söylenebilir.

- Nano materyal içerikli katkıların efektif güç, moment, efektif verim ve özgül yakıt tüketimi performans parametrelerini genelde olumlu yönde etkilediği söylenebilir.

- Nano materyal içerikli katkıların yakıt sistemi elemanlarına, motor elemanlarına ve egzoz gazı cihazlarına etkileri, ekonomiklik ve çevre etkileri açısından da değerlendirilmesi gerekir.

\section{SEMBOL VE KISALTMALAR}

\begin{tabular}{|c|c|}
\hline & \\
\hline $\mathrm{Al}_{2} \mathrm{O}_{3}$ & : Alüminyum oksit \\
\hline BD & : Biyodizel \\
\hline BDEX & : \% X etanol içeren biyodizel-etanol karışımı \\
\hline BDWX & : \% X su içeren biyodizel emülsiyon yakıtı \\
\hline $\mathrm{CaO}$ & : Kalsiyum oksit \\
\hline $\mathrm{CeO}_{2}$ & : Seryum oksit \\
\hline $\mathrm{Ce}-\mathrm{Zr}-\mathrm{O}_{2}$ & : Seryum zirkonyum oksit \\
\hline $\mathrm{Co}_{3} \mathrm{O}_{4}$ & : Kobalt oksit \\
\hline ÇTKNT & : Çok Tabakalı Karbon Nano Tüpler \\
\hline $\mathrm{D}$ & : Dizel yakıtı \\
\hline DBDXEY & : \% X biyodizel ve \% Y etanol içeren dizel-biyodizel-etanol karışımı \\
\hline DBDX & : \% X biyodizel içeren dizel-biyodizel karışımı \\
\hline
\end{tabular}




\begin{tabular}{|c|c|}
\hline DEX & : \% X etanol içeren dizel-etanol karışımı \\
\hline DWX & : \% X su içeren dizel emülsiyon yakıtı \\
\hline $\mathrm{E}$ & : Etanol \\
\hline $\mathrm{FeCl}_{3}$ & : Demir klorür \\
\hline $\mathrm{Fe}_{2} \mathrm{O}_{3}$ & : Demir II oksit \\
\hline $\mathrm{Fe}_{3} \mathrm{O}_{4}$ & : Demir III oksit \\
\hline GO & : Grafit oksit \\
\hline K & : Kerosin (havacılık yakıtı) \\
\hline KNT & : Karbon Nano Tüpler \\
\hline $\mathrm{MgO}$ & : Magnezyum oksit \\
\hline $\mathrm{MnO}_{2}$ & : Mangan dioksit \\
\hline $\mathrm{MnO}$ & : Mangan oksit \\
\hline $\mathrm{n}-\mathrm{Al}$ & : Nano alüminyum \\
\hline $\mathrm{n}-\mathrm{Al}-\mathrm{Mg}$ & : Nano alüminyum-magnezyum \\
\hline$n-B$ & : Nano bor \\
\hline $\mathrm{n}-\mathrm{Ce}$ & : Nano seryum \\
\hline $\mathrm{n}-\mathrm{Fe}$ & : Nano demir \\
\hline$n-G$ & : Nano grafit \\
\hline $\mathrm{n}-\mathrm{Ag}$ & : Nano gümüş \\
\hline $\mathrm{n}-\mathrm{Mn}$ & : Nano mangan \\
\hline $\mathrm{n}-\mathrm{Pt}$ & : Nano platin \\
\hline $\mathrm{n}-\mathrm{Si}$ & : Nano silikon \\
\hline $\mathrm{NiO}$ & : Nikel oksit \\
\hline $\mathrm{Rh}_{2} \mathrm{O}_{3}$ & : Rodyum oksit \\
\hline $\mathrm{TiO}_{2}$ & : Titanyum oksit \\
\hline $\mathrm{ZrO}_{2}$ & : Zirkonyum oksit \\
\hline $\mathrm{ZnO}$ & : Çinko oksit \\
\hline
\end{tabular}

\section{Kaynaklar}

[1] Murugesan, A., Umarani, C., Subramanian, R. ve Nedunchezhian, N., Bio-diesel as an alternative fuel for diesel engines-a review, Renewable and Sustainable Energy Reviews, 13, 653-662, (2009).

[2] Karabektas, M., Ergen, G. ve Hosoz, M., Effects of the blends containing low ratios of alternative fuels on the performance and emission characteristics of a diesel engine, Fuel, 112, 537-541, (2013).

[3] Dhar, A., Kevin, R. ve Agarwal A.K., Production of biodiesel from high-FFA neem oil and its performance, emission and combustion characterization in a single cylinder DICI engine, Fuel Processing Technology, 97, 118-129, (2012).

[4] Sharma, Y.C. ve Singh, B., Development of bio diesel from karanja, a tree found in rural India, Fuel, 87, 1740-1742, (2008).

[5] Chen, K.S., Lin, Y.C., Hsieh, L.T., Lin, L.F. ve Wu, C.C., Saving energy and reducing pollution by use of emulsified palm-biodiesel blends with bio-solution additive, Energy, 35, 2043-2048, (2010).

[6] Dantas, Neto A.A., Fernandes, M.R., Barros Neto, E.L., Castro Dantas, T.N. ve Moura, M.C.P.A., Alternative fuels composed by blends of non ionic surfactant with diesel and water: engine performance and emissions, Brazilian Journal of Chemical Engineering, 28, 3, 521-531, (2011). 
[7] Fu, W.B., Hou, L.Y., Wang, L.P. ve Ma, F.H., A study on ignition characteristics of emulsified oil containing flammable fuel, Fuel Processing Technology, 80, 921, (2003).

[8] Imdadul, H.K., Masjuki, H.H., Kalam, M.A., Zulkifli, N.W.M., Rashed, M.M., Rashedul, H.K., Monirul, I.M. ve Mosarof, M.H., A comprehensive review on the assessment of fuel additive effects on combustion behavior in CI engine fuelled with diesel biodiesel blends, RSC Advances, 5, 67541-67567, (2015).

[9] Shaafi, T., Sairam, K., Gopinath, A., Kumaresan, G. ve Velraj, V., Effect of dispersion of various nano additives on the performance and emission characteristics of a CI engine fuelled with diesel, biodiesel and blends-a review, Renewable and Sustainable Energy Reviews, 49, 563-573, (2015).

[10] Dreizin, E.L., Metal-based reactive nano materials, Progress in Energy and Combustion Science, 35, 141-167, (2009).

[11] Senthilraja, S., Karthikeyan, M. ve Gangadevi, R., Nanofluid applications in future automobiles: comprehensive review of existing data, Nano-MicroLetters, 2, 4, 306-310, (2010).

[12] Balamurugan, K., Tamilvanan, A., Anbarasu, M., Akil, M.S. ve Srihari, S., Nanocopper additive for reducing NOx emission in soya bean biodiesel-fuelled CI engine, Journal of Biofuels, 4, 1, 1-8, (2013).

[13] Sonawane, S., Patankar, K., Fogla, A., Puranik, B., Bhandarkar, U. ve Kumar, S.S., An experimental investigation of thermo-physical properties and heat transfer performance of Al2O3-Aviation turbine fuel nano fluids, Applied Thermal Engineering, 31, 2841-2849, (2011).

[14] Kao, M.J., Ting, C.C., Lin, B.F. and Tsung, T.T., Aqueous aluminum nano fluid combustion in diesel fuel, Journal of Testing and Evaluation, 36, 2, 1-5, (2008).

[15] Babu, K.R. ve Raja, R.B., Theoretical and experimental validation of performance and emission characteristics of nano additive blended diesel engine, International Journal of Research in Aeronautical and Mechanical Engineering, 3, 5, 18-31, (2015).

[16] Mohan, N., Sharma, M., Singh, R.C. ve Pandey, R.K., Performance study of diesel engine using nanofuel, International Journal of Advance Research and Innovation, 3, 4, 665-668, (2015).

[17] Saraee, H.S., Jafarmadar, S., Taghavifar, H. ve Ashrafi, S.J., Reduction of emissions and fuel consumption in a compression ignition engine using nanoparticles, International Journal of Environmental Science and Technology, 12, 2245-2252, (2015).

[18] Jung, H., Kittelson, D.B. ve Zachariah, M.R., The influence of a cerium additive on ultrafine diesel particle emissions and kinetics of oxidation, Combustion and Flame, 142, 276-288, (2005).

[19] Okuda, T., Schauer, J.J., Olson, M.R., Shafer, M.M., Rutter, A.P., Walz, K.A. ve Morschauser, P.A., Effects of a platinum-cerium bimetallic fuel additive on the chemical composition of diesel engine exhaust particles, Energy and Fuels, 23, 4974-4980, (2009).

[20] Mehta, R.N., Chakraborty, M. ve Parikh, P.A., Nano fuels: combustion, engine performance and emissions, Fuel, 120, 91-97, (2014).

[21] Venkatesan, S.P., Influence of aluminum oxide nano particle additive on performance and exhaust emissions of diesel engine, American-Eurasian Journal of Scientific Research, 10, 2, 88-92, (2015). 
[22] Raj, N.M., Gajendiran, M., Pitchandi, K. ve Nallusamy, N., Investigation on aluminium oxide nano particles blended diesel fuel combustion, performance and emission characteristics of a diesel engine, Journal of Chemical and Pharmaceutical Research, 8, 3, 246-257, (2016).

[23] Venkatesan, S.P. ve Kadiresh, P.N., Effects of nano-sized metal oxide additive on performance and exhaust emissions of CI engine, Applied Mechanics and Materials, 766-767, 389-395, (2015).

[24] Selvaganapthy, A., Sundar, A., Kumaragurubaran, B. ve Gopal, P., An experimental investigation to study the effects of various nano particles with diesel on DI diesel engine, ARPN Journal of Science and Technology, 3, 1, 112-115, (2013).

[25] Shafil, M.B., Daneshvar, F., Jahani, N. ve Mobini, K., Effect of ferro fluid on the performance and emission patterns of a four stroke diesel engine, Advances in Mechanical Engineering, ID529049, 5 pages, (2011).

[26] Sarvestany, N.S., Farzad, A., Bajestan, E.E. ve Mir, M., Effects of magnetic nano fluid fuel combustion on the performance and emission characteristics, Journal of Dispersion Science and Technology, 35, 1745-1750, (2013).

[27] Mahendravarman, R., Sivakumar, D.B. ve Sivakumar, P., Experimental study on performance and emission characteristics of a direct injection compression ignition engine with Fe3O4 nanoparticles, Advances in Natural and Applied Sciences, 10, 4, 139-144, (2016).

[28] Ramachandran, S.B., Ferrofluid-diesel blend, International Journal of Engineering and Applied Sciences, 2, 5, 92-94, (2015).

[29] Aalam, C.S., Saravanan, C.G. ve Premanand, B., Influence of Iron (II, III) oxide nano-particles fuel additive on exhaust emissions and combustion characteristics of CRDI system assisted diesel engine, International Journal of Advanced Engineering Research and Science, 2, 3, 23-28, (2015).

[30] Gumus, S., Ozcan, H., Ozbey, M. ve Topaloglu, B., Aluminum oxide and copper oxide nanodiesel fuel properties and usage in a compression ignition engine, Fuel, 163, 80-87, (2016).

[31] George, R.O., Franc, S., James, S.J., John, M. ve Sebastian, G., An experimental analysis on synergetic effect of multiple nanoparticle blended diesel fuel on CI engine, International Journal for Innovative Research in Science \& Technology, 1, 12, 151-156, (2015).

[32] Sungur, B., Topaloglu, B. ve Ozcan, H., Effects of nanoparticle additives to diesel on the combustion performance and emissions of a flame tube boiler, Energy, 113, 44-51, (2016).

[33] Ooi, J.B., Ismail, H.M., Swamy, V., Wang, X., Swain, A.K. ve Rajanren, J.R. Graphite oxide nanoparticles as diesel fuel additive for cleaner emission and lower fuel consumption, Energy and Fuels, 30, 2, 1341-1353, (2016).

[34] Lenin, M.A, Swaminathan, M.R. ve Kumaresan, G., Performance and emission characteristics of a DI diesel engine with a nano fuel additive, Fuel, 109, 362365, (2013).

[35] Keskin, A., Guru, M. ve Altiparmak, D., Influence of metallic based fuel additives on performance and exhaust emissions of diesel engine, Energy Conversion and Management, 52, 60-65, (2011).

[36] Guru, M., Karakaya, U., Altiparmak, D. ve Alicilar, A., Improvement of diesel fuel properties by using additives, Energy Conversion and Management, 43, 1021-1025, (2002). 
[37] Sajeevan, A.C. ve Sajith, V., Diesel engine emission reduction using catalytic nano-particles: an experimental investigation, Journal of Engineering, ID 589382, 9 pages, (2013).

[38] Selvan, V.A.M., Anand, R.B. ve Udaykumar, M., Effect of cerium oxide nano particle addition in diesel and diesel-biodiesel-ehanol blends on the performance and emission characteristics of a CI engine, ARPN Journal of Engineering and Applied Sciences, 4, 7, 1-6, (2009).

[39] Venkatesan, S.P., Kadiresh, P.N. ve Kumar, K.S. Experimental investigation of aqueous cerium oxide nano fluid blend in diesel engine, Advanced Materials Research, 938, 286-291, (2014).

[40] Zhang, J., Nazarenko, Y., Zhang, L., Calderon, L., Lee, K., Garfunkel, E., Schwander, S., Tetley, T.D., Chung, K.F., Porter, A.E., Ryan, M., Kipen, H., Lioy, P.J. ve Mainelis, G., Impacts of a nanosized ceria additive on diesel engine emissions of particulate and gaseous pollutants, Environmental Science and Technology, 47, 22, 13077-13085, (2013).

[41] Narasiman, V., Jeyakumar, S. ve Mani, M., Experimental investigation of DI diesel engine performance with oxygenated additive and SOME biodiesel, Bulletin of the JSME Journal of Thermal Science and Technology, 10, 1, 1-9, (2015).

[42] Aalam, C.S. ve Alagappan, N., Cerium oxide nano particles as addiditve with diesel fuel on DI diesel engine, International Journal of Innovative Research and Creative Technology, 1, 2, 215-219, (2015).

[43] Samuel, N. ve Shefeek, M.K., Performance and emission characteristics of a CI engine with cerium oxide nanoparticles as additive to diesel, International Journal of Science and Research, 4, 7, 672-676, (2015).

[44] Thirumal, B.J., Gunasekaran, E.J., Loganathan ve Saravanan, C.G., Emission reduction from a diesel engine fueled by cerium oxide nano-additives using SCR with different metal oxides coated catalytic converter, Journal of Engineering Science and Technology, 10, 11, 1404-1421, (2015).

[45] Venkatesan, S.P. ve Kadiresh, P.N. Influence of an aqueous cerium oxide nanofluid fuel additive on performance and emission characteristics of a compression ignition engine, International Journal of Ambient Energy, 37, 1, 64-67, (2016).

[46] Fangsuwannarak, K. ve Triratanasirichai, K., Effect of metalloid compound and bio solution additives on biodiesel engine performance and exhaust emissions, American Journal of Applied Sciences, 10, 10, 1201-1213, (2013).

[47] Aalam, C.S., Saravanan, C.G. ve Kannan, M., Experimental investigation on CRDI system assisted diesel engine fulled by diesel with nanotube, American Journal of Engineering and Applied Science, 8, 3, 380-389, (2015).

[48] Basha, S.J. ve Anand, R.B., An experimental study in a CI engine using nano additives blended water-diesel emulsion fuel, International Journal of Green Energy, 8, 3, 332-348, (2011).

[49] Mehta, R.N., Chakraborty, M. ve Parikh, P.A., Impact of hydrogen generated by splitting water with nano-silicon and nano-aluminum on diesel engine performance, International Journal of Hydrogen Energy, 39, 8098-8105, (2014).

[50] Singh, N. ve Bharj, R.S. Effect of CNT-emulsified fuel on performance, emission and combustion characteristics of four stroke diesel engine, International Journal of Current Engineering and Technology, 5, 1, 477-485, (2015). 
[51] Singh, N. ve Bharj, R.S., Experimental investigation on the role of indigenous carbon nanotube emulsified fuel in a four-stroke diesel engine, Journal of Mechanical Engineering Science, 230, 12, 2046-2059, (2016).

[52] Chaudhari, S.M., Thakare, S.V., Sontakke, K.G. ve Khodke, R.R., Effect of metal based additives on a CI engine fuelled with diesel and water, Journal of Emerging Technologies Innovative Research, 1, 7, 783-788, (2014).

[53] Mehregan, M. ve Moghiman, M., Numerical investigation of effect of nanoaluminum addition on $\mathrm{NOx}$ and $\mathrm{CO}$ pollutants emission in liquid fuels combustion, International Journal of Materials, Mechanics and Manufacturing, 2, 1, 60-63, (2014).

[54] Manikandan, R. ve Sethuraman, N., Experimental investigation of nano additive ceric oxide (CeO2)-ethanol blend on single cylinder four stroke diesel engine, International Journal of Recent Development in Engineering and Technology, 3, 2, 24-28, (2014).

[55] Ganesh, P.R. ve Reddy, K.H. Experimental investigation of performance and emission characteristics on a diesel engine using aqueous aluminium oxide and iron oxide nanoparticles as additives, Journal of Chemical and Pharmaceutical Sciences, 9, 3, 1747-1751, (2016).

[56] Basha, J.S. ve Anand, R.B. The influence of nano additive blended biodiesel fuel on the working characteristics of a diesel engine, Journal of the Brazilian Society of Mechanical Sciences and Engineering, 35, 257-264, (2013).

[57] Ganesh, D. ve Gowrishankar, G., Effect of nano-fuel additive on emission reduction in a biodiesel fuelled CI engine, IEEE, ID 978-1-4244-8165-1/11, 3453-3459, (2011).

[58] Banapurmath, N.R., Sankaran, R., Tumbal, A.V., Narasimhalu, T.N., Hunashyal, A.M. ve Ayachit, N.H., Experimental investigation on direct injection diesel engine fuelled with graphene, silver and multi walled carbon nano tubes biodiesel blended fuels, International Journal of Automotive Engineering and Technologies, 3, 4, 129-138, (2014).

[59] Banapurmath, N., Narasimhalu, T., Hunshyal, A., Sankaran, R., Rabinal, M.H., Ayachit, N. ve Kittur, R., Effect of silver nano-particle blended biodiesel and swirl on the performance of diesel engine combustion, International Journal of Sustainable and Green Energy, 3, 6, 150-157, (2014).

[60] Bhagwat, V.A., Navadagi, V. ve Dandavate, A., Experimental investigation of performance and emission characteristics of CI engine using graphene nanoparticles as an additive in biodiesel, International Engineering Research Journal, Special Issue 2, 4726-4732, (2015).

[61] Çelik, M., Combustion, performance and exhaust emission characteristics of organic based manganese addition to cotton methyl ester, Applied Thermal Engineering, 108, 1178-1189, 2016.

[62] Arockiasamy, P. ve Anand, R.B. Performance, combustion and emission characteristics of a DI diesel engine fuelled with nano particle blended jatropha biodiesel, Periodica Polytechnica Mechanical Engineering, 59, 2, 88-93, (2015).

[63] Balaji, G. ve Cheralathan, M., Influence of alumina oxide nanoparticles on the performance and emissions in a methyl ester of neem oil fuelled DI diesel engine, Thermal Science, Online-First Issue, 1-14.

[64] Kannan, G.R., Karvembu, R. ve Anand, R., Effect of metal based additive on performance emission and combustion characteristics of diesel engine fuelled with biodiesel, Applied Energy, 88, 3694-3703, (2011). 
[65] Amit ve Kumar, S., Impact on the performance of direct compression ignition engine by adding nano-particle in biodiesel, Journal of Material Science and Mechanical Engineering, 2, 7, 7-9, (2015).

[66] Yuvarajan, D. ve Ramanan, M.V. Effect of magnetite ferrofluid on the performance and emissions characteristics of diesel engine using methyl esters of mustard oil, Arabian Journal for Science and Engineering, 41, 2023-2030, (2016).

[67] Jeryrajkumar, L., Anbarasu, G. ve Elangovan, T., Effects on nano additives on performance and emission characteristics of calophyllim inophyllum biodiesel, International Journal of ChemTech Research, 9, 4, 210-219, (2016).

[68] Manibharathi, S., Annadurai, B. ve Chandraprakash, R., Experimental investigation of CI engine performance by nano additive in biofuel, International Journal of Science, Engineering and Technology Research, 3, 12, 3303-3307, (2014).

[69] Sajith, V., Sobhan, C.B. ve Peterson, G.P. Experimental investigations on the effects of cerium oxide nano particles fuel additives on biodiesel, Advances in Mechanical Engineering, ID 581407, 6 pages, (2010).

[70] Rajalingam, A., Jani, S.P., Kumar, A.S. ve Khan, A.A., The effect in neem oil biodiesel properties due to the blending of cerium oxide nanoparticles, International Journal for Research in Applied Science Engineering, Technology, 4, 2, 290-293, (2016).

[71] Balaji, G. ve Cheralathan, M., Effect of CNT as additive with biodiesel on the performance and emission characteristics of a DI diesel engine, International Journal of ChemTech Research, 7, 3, 1230-1236, (2015).

[72] Thulasi, G., Kandampalayam Ponnusamy, A., Rathanasamy, R., Palaniappan, S.K. ve Palanisamy, S.K. Reduction of harmful nitrogen oxide emission from low heat rejection diesel engine using carbon nanotubes, Thermal Science, 20, 4, 1181-1187, (2016).

[73] Tewari, P., Doijode, E., Banapurmath, N.R. ve Yaliwal, V.S., Experimental investigations on a diesel engine fuelled with multiwalled carbon nanotubes blended biodiesel fuels, International Journal of Emerging Technology and Advanced Engineering, 3, 3, 72-76, (2013).

[74] Basha, J.S. ve Anand, R.B. Role of nano additive blended biodiesel emulsion fuel on the working characteristics of a diesel engine, Journal of Renewable and Sustainable Energy, 3, 1-17, (2011).

[75] Anbarasu, A. ve Karthikeyan, A., Performance and emission characteristics of a diesel engine using cerium oxide nanoparticle blended biodiesel emulsion fuel, ASCE Journal of Energy Engineering, 1-7, (2015).

[76] Annamalai, M., Dhinesh, B., Nanthagopal, K., SivaramaKrishnan, P., Lalvani, J.I.J.R., Parthasarathy, M. ve Annamalai, K., An assessment on performance, combustion and emission behavior of a diesel engine powered by ceria nanoparticle blended emulsified biofuel, Energy Conversion and Management, 123, 372-380, (2016).

[77] Basha, J.S. ve Anand, R.B., Performance, emission and combustion characteristics of a diesel engine using carbon nano tubes blended jatropha methyl ester emulsions, Alexandria Engineering Journal, 53, 259-273, (2014).

[78] Venu, H. ve Madhavan, V., Effect of nano additives (titanium and zirconium oxides) and diethyl ether on biodiesel-ethanol fuelled CI engine, Journal of Mechanical Science Technology, 30, 5, 2361-2368, (2016). 
[79] Fangsuwannarak, K., Wanriko, P. ve Fangsuwannarak, T., Effect of bio-polymer additive on the fuel properties of palm biodiesel and on engine performance analysis and exhaust emission, Energy Procedia,100, 227-236, (2016).

[80] Kumar, V.N., Venkatesh, N.M. ve Alagumurthi, N., Influence of aluminum oxide $\mathrm{Al} 2 \mathrm{O} 3$ nano particles blended with waste cooking oil in the performance, emission and combustion characteristics on a DI diesel engine, Journal of Advanced Engineering Research, 3, 1, 66-71, (2016).

[81] Ramesh, D.K., Dhananjaya Kumar, J.L., Hemanth Kumar, S.G., Namith, V., Parashuram, B.J. ve Sharath, S., Study on effects of alumina nanoparticles as additive with poultry litter biodiesel on performance, combustion and emission characteristic of diesel engine, Materials Today, 338, 1-7, (2016).

[82] Aalam, C.S., Saravanan, C.G. ve Kannan, M., Experimental investigations on a CRDI system assisted diesel engine fuelled with aluminium oxide nanoparticles blended biodiesel, Alexandria Engineering Journal, 54, 3, 351-358, (2015).

[83] Karthikeyan, S., Elango, A., Silaimani, S.M. ve Prathima, A., Role of Al2O3 nano additive in GSO biodiesel on the working characteristics of a CI engine, Indian Journal of Chemical Technology, 21, 285-289, (2014).

[84] Ghogare, P. ve Kale, N.W., Experimental investigation on single cylinder diesel engine fuelled with cotton seed biodiesel blends with nano additives, International Conference on Electrical, Electronics, and Optimization Techniques, 17-22, (2016).

[85] Ghogare, P. ve Kale, N.W., Experimental investigation on single cylinder diesel engine fuelled with soya bean biodiesel blends with nano additives, International Journal of Pure and Applied Research in Engineering and Technology, 4, 9, 247-257, (2016).

[86] Sanjay, K.C., Shreyas, Pinto, V., Gafoor, S.S.A., Biju, T. ve Raju, K., Effect of alumina nanoparticles on the performance and emission characteristics of CI engine fuelled with lard oil methyl ester blends, American Journal of Materials Science, 6, 4A, 94-98, (2016).

[87] Prakash, P.J., Reddy, S.S.K., Kesavulu, P. ve Suresh, A., Experimental investigation on rhombus grooved piston with jatropha biodiesel and $\mathrm{Al} 2 \mathrm{O} 3$ nano fluid, International Journal of Engineering Research in Mechanical and Civil Engineering, 1, 6, 44-49, (2016).

[88] Aalam, C.S. ve Saravanan, C.G. Performance enhancement of common-rail diesel engine using $\mathrm{Al} 2 \mathrm{O} 3$ and $\mathrm{Fe} 3 \mathrm{O} 4$ nanoparticles blended biodiesel, International Research Journal of Engineering and Technology, 2, 5, 14001410, (2015).

[89] Jayanthi, P. ve Srinivasa, R.M. Effects of nanoparticles additives on performance and emissions characteristics of a DI diesel engine fuelled with biodiesel, International Journal of Advances in Engineering Technology, 9, 6, 689-695, (2016).

[90] Chandrasekaran, V., Arthanarisamy, M., Nachiappan, P., Dhanakotti, S. ve Moorthy, B., The role of nano additives for biodiesel and diesel blended transportation fuels, Transportation Research Part D, 46, 145-156, (2016).

[91] Kumar, C.V., Murugesan, A., Rajasekaran, T. ve Panneerselvam, N., Experimental investigation on the effects of nano additives on Mahuca Indica methyl ester-diesel fuel blends in diesel engine, Asian Journal of Research in Social Sciences and Humanities, 7, 1, 120-130, (2017). 
[92] Karthikeyan, S., Elango, A. ve Prathima, A., Performance and emission study on zinc oxide nano particles addition with pomoplion stearin wax biodiesel of CI engine, Journal of Scientific \& Industrial Research, 73, 187-190, (2014).

[93] Karthikeyan, S., Elango, A. ve Prathima, A., An environmental effect of GSO methyl ester with $\mathrm{ZnO}$ additive fuelled marine engine, Indian Journal of GeoMarine Sciences, 43, 4, 564-570, (2014).

[94] Silambarasan, R. ve Senthil, R., Effects of nano additives on performance and emission characteristics of a diesel engine fueled with Annona methyl ester, Biofuels, 1-7, (2016).

[95] Karthikeyan, S., Elango, A. ve Prathima, A., Diesel engine performance and emission analysis using canola oil methyl ester with the nano sized zinc oxide particles, Indian Journal of Engineering Material Sciences, 21, 83-87, (2014).

[96] Santhanamuthu, M., Chittibabu, S., Tamizharasan, T. ve Mani, T.P., Evaluation of CI engine performance fuelled by diesel-polanga oil blends doped with iron oxide nano particles, International Journal of ChemTech Research, 6, 2, 12991308, (2014).

[97] Keskin, A., Guru, M. ve Altiparmak, D., Biodiesel production from tall oil with synthesized $\mathrm{Mn}$ and $\mathrm{Ni}$ based additives: effects of the additives on fuel consumption and emissions, Fuel, 86, 1139-1143, (2007).

[98] Manibharathi, S., Chandraprakash, R., Annadurai, B. ve Titus, R., Experimental investigation of $\mathrm{CI}$ engine performance and emission characteristics by effect of nano fuel additives in pongamia pinnata biodiesel, International Journal for Scientific Research Development, 3, 1, 1043-1047, (2015).

[99] Ingle, S.S., Nandedkar, V.M. ve Joshi, K.G., Experimental investigation of palm biodiesel with nanomaterial as a fuel additive on performance and emission of diesel engine, International Journal of Innovative Research in Science, Engineering and Technology, 5, 11, 19869-19879, (2016).

[100] Karthikeyan, S., Elango, A. ve Prathima, A., The effect of cerium oxide additive on the performance and emission characteristics of a CI engine operated with rice bran biodiesel and its blends, International Journal of Green Energy, 13, 3, 267-273, (2016).

[101] Ramarao, K., Rao, C.J. ve Sreeramulu, D., The experimental investigation on performance and emission characteristics of a single cylinder diesel engine using nano additives in diesel and biodiesel, Indian Journal of Science and Technology, 8, 29, 1-9, (2015).

[102] Bafghi, A.A.T., Bakhoda, H. ve Chegeni, F.K., Effects of cerium oxide nanoparticle addition in diesel and diesel-biodiesel blends on the performance characteristics of a CI engine, International Journal of Mechanical, Aerospace, Industrial, Mechatronic and Manufacturing Engineering, 9, 8, 1507-1512, (2015).

[103] Karthikeyan, S., Elango, A., Silaimani, S.M. ve Prathima, A., Performance, combustion end emission characteristic of a marine engine running on grape seed oil biodiesel with nano additive, Indian Journal of Geo-Marine Sciences, 43, 12, 2315-2319, (2014).

[104] Sathiyamoorthi, R., Puviyarasan, M., Kumar, B.B. ve Joshua, D.B. Effect of $\mathrm{CeO} 2$ nano additive on performance and emissions of diesel engine fuelled by neem oil-biodiesel, International Journal of Chemical Sciences, 14, 2, 473484, (2016). 
[105] Sajith, V. ve Mohamed Jihad, P.M., Development of stable cerium zirconium mixed oxide nano particle additive for emission reduction in biodiesel blends, Journal of Engineering and Technology, 4, 2, 23-34, (2015).

[106] D’Silva, R., Vinoothan, K., Binu, K.G., Thirumaleshwara, B. ve Raju, K., Effect of titanium dioxide and calcium carbonate nanoadditives on the performance and emission characteristics of CI engine, Journal of Mechanical Engineering and Automation, 6, 5A, 28-31, (2016).

[107] Fangsuwannarak, K. ve Triratanasirichai, K., Improvements of palm biodiesel properties by using nano-TiO2 additive, emissions and engine performance, Romanian Review Precision Mechanics, Optics \& Mechatronics, 43, 111-118, (2013).

[108] Prabhu, L., Kumar, S.S., Andrerson, A. ve Rajan, K., Investigation on performance and emission analysis of $\mathrm{TiO} 2$ nanoparticle as an additive for biodiesel blends, Journal of Chemical and Pharmaceutical Sciences, Special Issue 7, 408-412, (2015).

[109] Mirzajanzadeh, M., Tabatabaei, M., Ardjmand, M., Rashidi, A., Ghobadian, B., Barkhi, M. ve Pazouki, M., A novel soluble nano-catalysts in diesel-biodiesel fuel blends to improve diesel engines performance and reduce exhaust emissions, Fuel, 139, 374-382, (2015).

[110] Karthikeyan, S. ve Prathima, A., Emission analysis of the effect of doped nanoadditives on biofuel in a diesel engine, Energy Sources Part A, 38, 24, 3702-3708, (2016).

[111] Ghafoori, M., Ghobadian, B., Najafi, G., Layeghi, M., Rashidi, A. ve Mamat, R., Effect of nano-particles on the performance and emission of a diesel engine using biodiesel-diesel blend, International Journal of Automotive and Mechanical Engineering, 12, 3097-3108, (2015).

[112] Madhukrishnaa, N., Sivakumar, D.B., Arulmozhi, M., Chandrasekar, M. ve Senthilkumar, T., Performance and emission analysis of cotton seed oil biodiesel with alumina nanoparticle additive, International Journal of Research Science and Engineering, 1-6, (2016).

[113] Shaafi, T. ve Velraj, R., Influence of alumina nano particles, ethanol and isopropanol blend as additive with diesel soybean biodiesel blend fuel: combustion, engine performance and emissions, Renewable Energy, 80, 655663, (2015).

[114] Selvan, V.A.M., Anand, R.B. ve Udayakumar, M., Effect of cerium oxide nano particles and carbon nano tubes as fuel-borne additives in diesterol blends on the performance, combustion and emission characteristics of a variable compression ratio engine, Fuel, 130: 160-167, (2014).

[115] Jose, M. and Anand, R.B., Studies on compression ignition engine to establish effects of injection pressure, compression ratio and nano additives-a review, International Journal of Advances in Engineering Research, 2, 4, 1-17, (2011).

[116] Ghogardare, S.V. ve Hudgikar, S.R.K., Review on performance and emission characteristics of CI engine by using nanoparticles with blended biodiesel in diesel fuel, International Engineering Research Journal, 2, 6, 2120-2122, (2016).

[117] Soni, G.S., Rathod, P.P. ve Goswami, J.J., Performance and emission characteristics of CI engine using diesel and biodiesel blends with nanoparticles as additive-A review study, International Journal of Engineering Development and Research, 3, 4, 879-884, (2015). 
[118] Khond, V.W. ve Kriplani, V.M., Effect of nano fluid additives on performances and emissions of emulsified diesel and biodiesel fueled stationary CI engine: A comprehensive review, Renewable and Sustainable Energy Reviews, 59, 13381348, (2016). 